\title{
Modeling of Symbiotic Bacterial Biofilm Growth with an Example of the Streptococcus-Veillonella sp. System
}

\author{
Dianlei Feng $^{1}$ (D) Insa Neuweiler ${ }^{1} \cdot$ Regina Nogueira $^{2} \cdot$ Udo Nackenhorst $^{3}$
}

Received: 15 November 2019 / Accepted: 11 March 2021 / Published online: 24 March 2021

(c) The Author(s) 2021

\begin{abstract}
We present a multi-dimensional continuum mathematical model for modeling the growth of a symbiotic biofilm system. We take a dual-species namely, the Streptococcus-Veillonella sp. biofilm system as an example for numerical investigations. The presented model describes both the cooperation and competition between these species of bacteria. The coupled partial differential equations are solved by using an integrative finite element numerical strategy. Numerical examples are carried out for studying the evolution and distribution of the bio-components. The results demonstrate that the presented model is capable of describing the symbiotic behavior of the biofilm system. However, homogenized numerical solutions are observed locally. To study the homogenization behavior of the model, numerical investigations regarding on how random initial biomass distribution influences the homogenization process are carried out. We found that a smaller correlation length of the initial biomass distribution leads to faster homogenization of the solution globally, however, shows more fluctuated biomass profiles along the biofilm thickness direction. More realistic scenarios with bacteria in patches are also investigated numerically in this study.
\end{abstract}

Keywords Biofilm model - Symbiotic system - Streptococcus-Veillonella . Numerical simulation $\cdot$ Heterogeneous distribution

Dianlei Feng

feng@hydromech.uni-hannover.de

1 Institute of Fluid Mechanics and Environmental Physics in Civil Engineering, Leibniz Universität Hannover, Appelstraße 9a, 30167 Hannover, Germany

2 Institute for Sanitary Engineering and Waste Management, Gottfried Wilhelm Leibniz Universität Hannover, Welfengarten 1, 30163 Hannover, Germany

3 Institute of Mechanics and Computational Mechanics, Leibniz Universität Hannover, Appelstraße 9a, 30167 Hannover, Germany 


\section{Introduction}

More than $90 \%$ of microbes live in biofilms which can be defined as "assemblages of bacterial cells attached to a surface and enclosed in an adhesive matrix secreted by the cells" (Madigan 2012). A biofilm in natura is usually found as a multi-component, multi-species, heterotopic matter with multi-phase properties. Competition and cooperation among species of bacteria are normally involved in multi-species biofilm systems (Yang et al. 2011). A biological system is symbiotic when cooperation happens between two different organisms.

Mathematical and numerical modeling is a powerful tool for understanding both the physical and bio-chemical processes during the formation and development of the biofilms. Many modeling strategies and models have been developed for describing biofilm processes. However, mathematical modeling of symbiotic biofilm systems has not been well studied. Modeling a symbiotic biofilm system naturally requires the consideration of a multi-species biofilm problem which is usually challenging, especially for multi-dimensional scenarios.

Several studies on modeling multi-dimensional multi-species biofilm system have been carried out in the literature. Noguera et al. (1999) presented a Cellular Automaton (CA) model for modeling a dual-species (D. vulgaris-M. formicicum) biofilm system. Recently, Tang and Liu (2017) developed a multi-species multi-dimensional CA model to study syntrophic and dissimilatory metal reducing bacterial bioiflm system. In their study, a syntrophic ecological system is considered. Martin et al. (2017) studied a dual-species ( $S$. gordonii-P. gingivalis) oral biofilm system with a further developed CA model. Three models on the relationship between these two species of bacteria, namely independence for substrates, competition for substrate, and inhibition of one to another, are compared in their study. Individual-based Modeling (IbM) have also been used for studying various multi-species biofilm problems, such as modeling ammonium oxidizer bacteria (AOB) and nitrite oxidizer bacteria (NOB) system (Picioreanu et al. 2004), modeling dormant cell formation (Chihara et al. 2015) process, investigation of denitrifying bacteria - sulfate reducing bacteria-methanogens biofilm systems (Martin et al. 2015) and so forth. These models are not continuum and are usually categorized as the so-called discrete element based models (Noguera et al. 1999; Fujikawa 1994; Kreft et al. 2001; Picioreanu et al. 1998; Wimpenny and Colasanti 1997).

Although the reaction model presented in this paper can also be applied with discrete element based models, we present our model within the framework of continuum biofilm models which are fully described by partial differential equations (Alpkvist and Klapper 2007; Cogan 2004; Duddu et al. 2009; Eberl et al. 2001; Klapper and Dockery 2002; Lindley et al. 2012; Wanner and Gujer 1986; Zhang et al. 2008b). Alpkvist and Klapper (2007) presented a multi-dimensional multi-species biofilm model and applied it for studying an autotrophs-heterotrophs-inert bifilm system. The growth of biofilm is modeled as an advective movement of a potential flow due to the production or reduction of biomass. Instead of modeling the biofilms grow advectively, Rahman et al. (2015) presented a multi-species multi-dimensional continuum diffusion-reaction model. A cross-diffusion process is additionally introduced into the model to overcome the internal over-mixing problem. The over-mixing of different species of biomass 
is a common flaw in many diffusion-reaction continuum models as well as some discrete element based models for multi-species multi-dimensional biofilm modeling (Tang and Valocchi 2013). Since the advection-reaction biofilm model presented by Alpkvist and Klapper (2007) does not involve physical diffusion, it is expected to be less diffusive, although the numerical dissipation is not avoidable. However, when the reactions become complex, whether locally homogenized biomass distribution will be obtained or not is still an open question. We look into such a problem by studying the presented symbiotic biofilm system with an advection-reaction type model, and one goal of this study is to investigate the solution behaviors of the model.

We take an oral dual-species, namely the Streptococcus-Veillonella sp. biofilm system (Chalmers et al. 2008), as an example of modeling symbiotic biofilm systems. Both Streptococcus sp. and Veillonella sp. play vital roles in the formation of dental biofilms. Dental bacterial biofilms are often of special interested due to their roles in the treatments of dental implants and diseases (Paquette et al. 2006; Kommerein et al. 2017). Different from many other types of biofilms, the bacteria that form the dental biofilms need salivary glycoproteins to attach to the teeth surface. Meanwhile, saliva is also the main nutrient source for the microbes in oral cavities (Chalmers et al. 2008; Chalmers 2008). Among the Streptococcus group bacteria, Streptococcus gordonii is a well-known Gram-Positive commensal bacterium in oral cavities which can cause caries and demineralization of teeth (Nascimento et al. 2009). Streptococcus gordonii is also the first batch of bacteria that attach to the teeth surface and is known as a species of early colonizers (Kolenbrander et al. 2010). The early colonizers can build up a foundation for the later colonizers to attach to the biofilm structure. This makes them important for studying oral biofilms.

Similar to the $S$. gordonii, Veillonella sp. is also a group of early colonizers which congregate with S. gordonii (Periasamy and Kolenbrander 2010). It has been discovered that the Veillonella sp. cannot grow alone in saliva. However, it colonizes when there is Strptococcus sp. (e.g. S. gordonii) coexisting in the system (Chalmers et al. 2008). Similar behavior has also been observed in Kara et al. (2007), Mashima and Nakazawa (2015). A biological interpretation of such phenomenon is that the Veillonella sp. cannot utilize the carbon source in saliva directly but can ferment the metabolic production of $S$. gordonii, namely lactic acid, instead (Periasamy and Kolenbrander 2010). It is worth noting that the lactic acid not only provides a carbon source for the growth of the Veillonella sp. but also causes a decrease of the $\mathrm{pH}$ in the system. It has been experimentally observed that the Veillonella sp. can tolerate more acid than the S. gordonii (Bradshaw and Marsh 1998). The presence of the Veillonella which utilizes the lactic acid may thus be beneficial to the growth of the $S$. gordonii indirectly.

In this study, we develop a mathematical model for the symbiotic S. gordoniiVeillonella biofilm system under the hypothesis that the lactic acid has a negative influence on the growth of $S$. gordonii and thus the symbiotic biofilm system studied in this paper is essentially a mutualistic ecological system. The flow velocity in oral cavities is known to be very small and does not have much impact on the growth of the $S$. gordonii biofilm within a typical characteristic period (12h-24h) (Rath et al. 2017). The mathematical model developed by Alpkvist and Klapper (2007) has been validated for the $S$. gordonii biofilm (Rath et al. 2017; Feng et al. 2018). Many studies have reported that the Streptococcus sp. dominates in a multi-species biofilm, which 
consists of the Streptococcus sp. and Veillonella sp. (Chalmers et al. 2008; Periasamy and Kolenbrander 2010; Kommerein et al. 2017). For this reason, we develop the symbiotic biofilm model based on the advection-reaction biofilm growth model.

The model presented in this paper can be used for quantitatively understanding of the Streptococcus-Veillonella symbiotic biofillm system and in general can be modified to model a variety of biofilm systems with similar bio-chemical properties. To the best of our knowledge, a continuum model for such kind of symbiotic biofilm systems has not been well developed. This study may shed light on understanding the numerical behaviors of multi-dimensional multi-species continuum (advection-reaction) biofilm models which involve complex reactions.

This paper is structured as follows. Details of the model will be presented in Sect. 2. Numerical results are presented and investigated in Sect. 3. The symbiotic biofilm model is highly non-linear. We observed a homogenized numerical solution of biomass at a later time. To better understand the solution behaviors, we investigated how the initial condition (see Sect. 3.2) and the symbiotic reaction relationship (see Sect. 3.4) influence the homogenization of the solutions. In Sect. 4, the results are summarized and discussed. Further detailed information regarding the dimensionless form of the governing equations, as well as the corresponding numerical method, are presented in Appendices A and B.

\section{Mathematical Model}

\subsection{Model Assumptions}

The Streptococcus-Veillonella biofilm system is illustrated in Fig. 2. Two biomass components, namely the S. gordonii and V. PK1910 interact with each other in a mutualistic relationship. Especially, $S$. gordonii produces lactic acid, which plays a vital role in the chain of the symbiotic bio-chemical process is modeled explicitly. Despite the saliva cannot function as a carbon source for the Veillonella sp., saliva still may provide other nutrients for the growth of both species due to its complex mixture substance property. Therefore, a competition of the saliva between $S$. gordonii and Veillonella sp. is considered in the model. On the other hand, Veillonella sp. helps to prevent the environment from being too acid, and thus benefits the growth of $S$. gordonii. We develop the mathematical model based on the following assumptions:

- A layer of biomass with a finite thickness has been presented as an initial condition. The model does not capture the process in which individual bacteria grow into a biofilm. On the other hand, the growth process after forming an initial layer of biofilm is modeled.

- Two species of bacteria, namely the Streptococcus sp., and Veillonella sp. are considered in the model. The volume fractions of the bacteria sum to one inside of the biofilm, which implies that the pore space and EPS are not explicitly modeled (Alpkvist and Klapper 2007). 
- Saliva is considered as a common nutrient source consumed by both species of bacteria. However, the growth of Veillonella is limited by both the nutrient and the lactic acid, which is produced by Streptoccus sp. (Chalmers et al. 2008).

- The concentration of hydrogen is not modeled explicitly, but the concentration of the lactic acid is considered instead as an indicator for it (Gordeeva et al. 2017).

- The fluid-structure interaction process is not considered. This assumption is based on our previous experimental observations of the formation process of $S$. gordonii (Rath et al. 2017).

- No new void spaces of bacteria can develop during the growth of the biofilm. In other words, the biofilm domain is simply connected (see Fig. 1).

- The nutrients in saliva are fully mixed with a distance above the biofilm. A Dirichlet boundary condition of the nutrients thus exists (Picioreanu et al. 2006).

- The growth velocity is irrotational (Klapper and Dockery 2002), and different species of bacteria have the same advection velocity (Alpkvist and Klapper 2007).

- Diffusion coefficients are constant both inside and outside of the biofilm (Alpkvist and Klapper 2007).

To make the model simple, we introduced assumptions more than necessary. One can include the EPS as an additional biomass component in the model, as in Xavier et al. (2005). It is also convenient to assign heterogeneous modeling parameters, such as diffusion coefficient, density, or even growth rate in the model (Fig. 2). The pH can also be modeled by slightly extending the model, as presented in Khassehkhan and Eberl (2008).

The main limitation of the presented model is the requirement of being simply connected (biofilm domain) during biofilm growth. This is mostly due to the difficulty of enforcing proper potential (or pressure) boundary conditions for the newly generated biofilm-fluid surface. Such a problem may come up when trying to model the merge of different colonies. As illustrated in Fig. 1b, when there is a new biofilm-fluid interface $\left(\Gamma_{2}\right)$ generated during the merge of colonies, it is not straightforward to enforce boundary conditions of the potential on $\Gamma_{1}$ and $\Gamma_{2}$. On the other hand, the model can be used to model the merge of different colonies if only the scenario, as shown in Fig. 1a, occurs. Modeling the merge of colonies is beyond the aim of this paper. For the readers interested in this topic, we refer to the three-dimension numerical study in Alpkvist and Klapper (2007).

\subsection{Model Domain}

To build up the model based on the above assumptions, the mass balance of each substrate and component of biomass in the biofilm is considered. Eventually, the mathematical model, which is essentially a free boundary problem, consists of five partial differential equations (PDEs) including four coupled nonlinear time dependent advection-diffusion-reaction (or advection-reaction) equations and a Poisson's equation.

Taking a 2D model as an example, the biofilm is modeled within a computational domain of $\Omega=\{\mathbf{x}=(x, z): 0 \leq x \leq W, 0 \leq z \leq H\}$ with the corresponding boundary $\partial \Omega$. The top border is denoted as $\Gamma_{h t}$ and the other sides are denoted by 


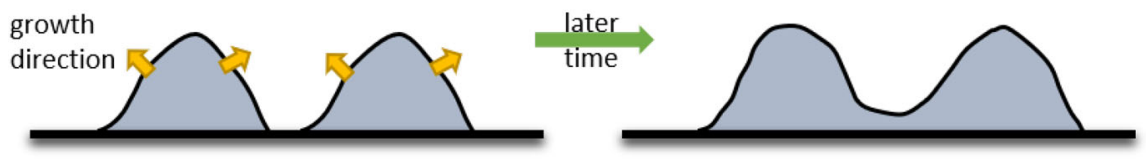

(a) simply connected

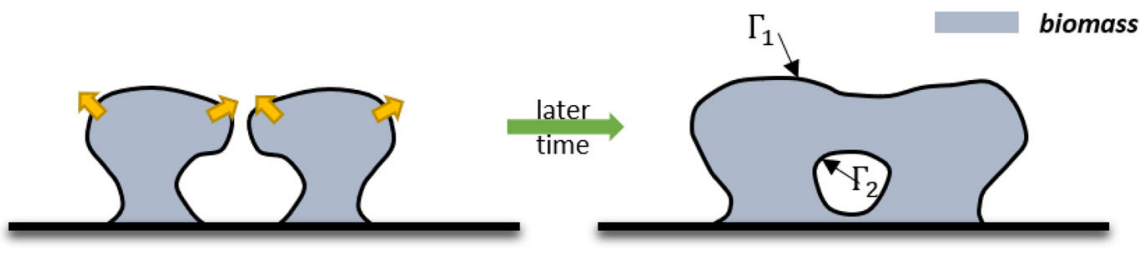

(b) not simply connected

Fig. 1 Typical biofilm patterns generated during the merge of two colonies

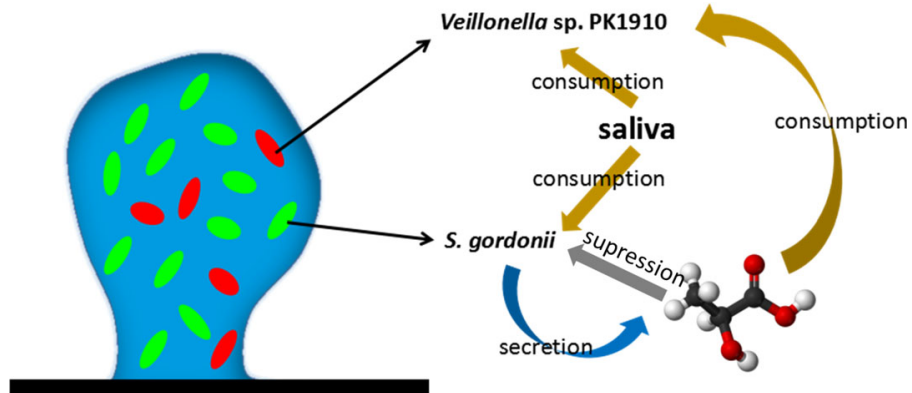

lactic acid

Fig. 2 Illustration of the symbiotic biofilm system. The Veillonella sp. PK1910 and Streptococcus gordonii are taken as example species of the bacteria in the illustration. Both species of bacteria compete for the nutrients in saliva. The Streptococcus gordonii produces lactic acid, which is a carbon source for Veillonella sp. The lactic acid also prevents the growth of gordonii

$\Gamma_{s 1}=\partial \Omega \nsupseteq \Gamma_{h t}$. The outward-pointing normal vectors of $\Gamma_{s 1}$ and $\partial \Omega$ are denoted as $\mathbf{n}_{s 1}$ and $\mathbf{n}_{s 2}$ respectively. A sub-domain $\mathrm{B}_{t} \in \Omega$ refers to the biofilm domain above which a bulk fluid domain $\mathrm{F}_{t}$ is assumed. The biofilm-fluid interface $\Gamma_{i n t}=B_{t} \cap F_{t}$, which is a free boundary, moves over time induced by the growth of the biofilm.

\subsection{Mass Balance of Biomass}

The growth of both $S$. gordonii and Veillonella are modeled as advective movements driven by the production and reduction of biomass inside of the biofilm. Writing the mass balance equations of $S$. gordonii and Veillonella sp. in terms of their volume 
Fig. 3 Two dimensional illustration of the computational domains of the symbiotic biofilm model

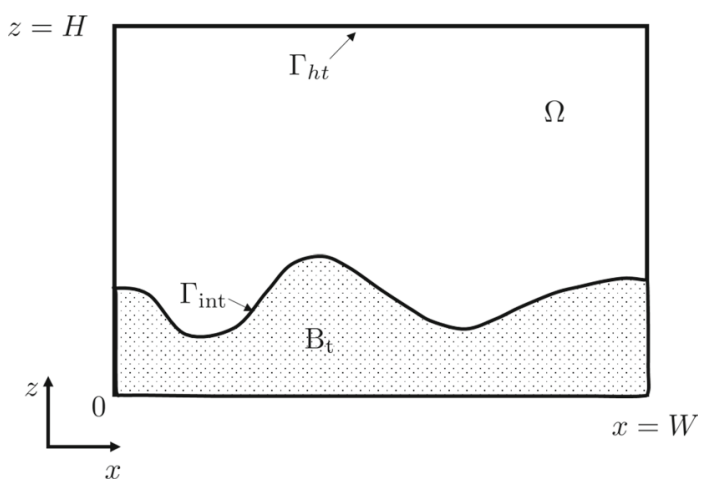

fraction $\vartheta$ reads

$$
\begin{array}{ll}
\frac{\partial \vartheta_{1}}{\partial t}+\nabla \cdot\left(\mathbf{u} \vartheta_{1}\right)=\frac{g_{1}}{\rho_{s}}, & \mathbf{x} \in \Omega, \\
\frac{\partial \vartheta_{2}}{\partial t}+\nabla \cdot\left(\mathbf{u} \vartheta_{2}\right)=\frac{g_{2}}{\rho_{v}}, & \mathbf{x} \in \Omega,
\end{array}
$$

where $\mathbf{u}\left[L T^{-1}\right]$ denotes the growth velocity of the biofilm, indexes "1" and "2" are used to refer physical quantities of the $S$. gordonii and Veillonella bacteria respectively and, $\rho_{s}$ and $\rho_{v}$ denote the density of the corresponding biomass. In Eq. (1), $g$ refers to the mass production/reduction rate of the biomass. A no-flow boundary is applied for both Eqs. (1a) and (1b)

$$
\mathbf{n}_{s 2} \cdot\left(\mathbf{u} \vartheta_{i}\right)=0, \quad(i=1,2), \quad \mathbf{x} \in \partial \Omega
$$

It has been experimentally found that the $S$. gordonii can tolerate less acid than the Veillonella sp. and the Veillonella sp. may become dominant even in the environment with $\mathrm{pH}<5.0$ (Bradshaw and Marsh 1998). The growth of $S$. gordonii is sensitive to the acid and, on the other hand, Veillonella can grow in a small $\mathrm{pH}$ environment. Therefore, we only model the negative influence of lactic acid on the growth of $S$. gordonii. The mass production process of $S$. gordonii is limited by the concentration of saliva $\left(s_{1}\right)$ and suppressed by the lactic acid $\left(s_{2}\right)$

$$
g_{1}=\vartheta_{1} \rho_{s} \mu_{1} \frac{s_{1}}{k_{11}+s_{1}} \frac{k_{l}}{k_{l}+s_{2}} .
$$

In Eq. (3), $\mu_{1}\left[T^{-1}\right]$ and $k_{11}\left[M L^{-3}\right]$ refer to the maximum growth rate and the half-rate constant of the $S$. gordonii in the Monod kinetic respectively. We introduce a parameter $k_{l}\left[M L^{-3}\right]$ for modeling the suppression process induced by the lactic acid. A benefit of choosing $g_{1}$ in the form as Eq. (3) is, when $s_{2} \rightarrow+\infty, g_{1} \rightarrow 0$. This means the growth of the $S$. gordonii stops when the concentration of the lactic acid is infinitely large. 
The growth of Veillonella is limited by both the concentration of the nutrients in saliva $s_{1}$ and the concentration of the lactic acid $s_{2}$

$$
g_{2}=\vartheta_{2} \rho_{v} \mu_{2} \frac{s_{1}}{k_{21}+s_{1}} \frac{s_{2}}{k_{22}+s_{2}} .
$$

In Eq. (4), $\mu_{2}\left[T^{-1}\right]$ denotes the maximum growth rate of Veillonella and $k_{21}\left[M L^{-3}\right]$ and $k_{22}\left[M L^{-3}\right]$ are the half-rate constant in the Monod kinetic.

\subsection{Mass Balance of Saliva and Lactic Acid}

The chemical reactions of components in the saliva environment are very complex, and the corresponding reaction parameters are mostly unknown. Instead of describing detailed chemical reactions of all involved chemical components in saliva, we consider the saliva itself as one substance demanded by both species of bacteria. Since the time scale of the transport process of the substrate (solutions) is much smaller than the time scale of the biofilm growth (Picioreanu et al. 2000), the mass balance of the saliva is modeled as a stationary diffusion-reaction process as

$$
-D_{1} \nabla^{2} s_{1}=r_{11}+r_{12}, \quad \mathbf{x} \in \Omega,
$$

where $r_{11}$ and $r_{12}\left[\mathrm{ML}^{-3} T^{-1}\right]$ denote the saliva (nutrients in saliva) consumption rates by $S$. gordonii and Veillonella sp. respectively, and $D_{1}\left[L^{2} T^{-1}\right]$ denotes the diffusion coefficient of the saliva. As a remark, one can also write (5) as a time-dependent equation (Wanner and Reichert 1996).

The nutrient in saliva comes from the outside of the modeling domain $\Omega$. We are interested in the symbiotic behavior of bacteria in a batch system with sufficient supplying nutrients in this study. For this reason, we consider a specific batch system with a controlled concentration of nutrients. Accordingly, we apply a boundary condition where a constant value $\bar{s}_{1}$ is set at the top of the computational domain $\Gamma_{h t}$ (as shown in Fig. 3)

$$
s_{1}=\bar{s}_{1}, \quad \mathbf{x} \in \Gamma_{h t} .
$$

As a remark, if the nutrient concentration $s_{1}$ is not controlled in the application, one needs to modify Eq. (5) by involving the time-dependent term and also apply a no-flow boundary at the top of the computational domain. A no-flow boundary is applied on the other borders of the computational domain as

$$
\mathbf{n}_{s 1} \cdot \nabla s_{1}=0, \quad \mathbf{x} \in \Gamma_{s 1},
$$

where $\mathbf{n}_{s 1}$ denotes the normal vector of $\Gamma_{s 1}$.

The reaction term corresponding to the consumption of saliva by $S$. gordonii is described by using the Monod kinetics and reads

$$
r_{11}=-\frac{1}{Y_{s 1}} g_{1}
$$


where $Y_{S 1}[-]$ denotes the corresponding yield of the $S$. gordonii by consuming rate of the saliva. Similarly, the consumption rate of the saliva due to the Veillonella reads

$$
r_{12}\left(s_{1}, s_{2}, \vartheta_{2}\right)=-\frac{1}{Y_{v 1}} g_{2},
$$

where $Y_{v 1}[-]$ is the yield of the Veillonella.

The lactic acid which is secreted by the $S$. gordonii accumulates over time in the system. If a batch system is considered, the lactic acid is produced inside of the biofilm domain $\mathrm{B}_{t}$ and stays in the system. In this case, the mass balance of the lactic acid cannot be modeled as a quasi-steady state since the absence of the Dirichlet boundary condition. For these reasons, the mass balance of the acid has to be modeled as a time dependent process. The mass transport of acid is diffusion-reaction dominated. Therefore, one can simplify the model by neglecting the advection process and achieve comparable, accurate results. Moreover, the production and consumption of the lactic acid by $S$. gordonii and Veillonella sp. are also considered respectively. The mass balance of the lactic acid reads

$$
\frac{\partial s_{2}}{\partial t}+\nabla \cdot\left(\mathbf{u} s_{2}\right)-D_{2} \nabla^{2} s_{2}=r_{21}+r_{22}, \quad \mathbf{x} \in \Omega,
$$

where $D_{2}\left[L^{2} T^{-1}\right]$ is the diffusion coefficient of the lactic acid. $r_{21}\left[M L^{-3} T^{-1}\right]$ and $r_{22}\left[M L^{-3} T^{-1}\right]$ describe the production of the lactic acid by $S$. gordonii and the consumption rate of the acid by the Veillonella respectively. As a remark, the advection term in (10) is negligible. The Péclet number for the corresponding process has an order of magnitude $10^{-4}$ (estimated by using parameters in Table 1). We solve all time-dependent equations simultaneously using the stabilized space-time finite element method (see Appendix B). Keeping the advection term in (10) makes it more convenient for the numerical implementation.

S. gordonii grows by consuming saliva and producing lactic acid at the same time. Thus the reaction term $r_{21}$ can be written as

$$
r_{21}=\lambda g_{1}
$$

where $\lambda[-]$ is a parameter which describes the mass production rate of acid by unit mass of S. gordonii. Only the Veillonella sp. in the system consumes lactic acid, therefore, the reaction kinetic can be written as

$$
r_{22}\left(s_{1}, s_{2}, \vartheta_{2}\right)=-\frac{1}{Y_{v 2}} g_{2},
$$

where $Y_{v 2}$ refers to the yield of Veillonella by consuming the lactic acid. A no-flow boundary is applied on $\partial \Omega$ for Eq. (10) as

$$
\mathbf{n}_{s 2} \cdot\left(\mathbf{u} \nabla s_{2}-D_{2} \nabla s_{2}\right)=0, \quad \mathbf{x} \in \partial \Omega
$$

where $\mathbf{n}_{s 2}$ denotes the normal vector on $\partial \Omega$. 
We applied a no-flow boundary condition in the saliva's mass balance equation. Such a boundary condition implies that a batch system is modeled. For flow chamber systems, one may also invoke a Dirichlet boundary for the acid (see later discussions in Sect. 3.1.2). Alternatively, one can apply a diffusive boundary layer (Feng et al. 2017) which essentially is a Robin boundary condition (Ghasemi et al. 2018; Wanner and Gujer 1986) for allowing the acid to leave the domain.

\subsection{Potential Equation for the Movement of the Biofilm}

We model the movement of the biofilm as a potential flow driven by the production of the biomass inside of the biofilm following (Klapper and Dockery 2002; Alpkvist and Klapper 2007). It is assumed that the two species of bacteria compose the whole biofilm. Therefore, the volume fractions of the $S$. gordonii and Veillonella sum up to one in the biofilm domain $\mathrm{B}_{t}$ as

$$
\sum_{i=1}^{2} \vartheta_{i}=1, \quad \mathbf{x} \in \partial B_{t} .
$$

Following the previous potential flow assumption, the biofilm growth velocity is described as the gradient of the potential $\Phi$. Substituting Eqs. (1a) and (1b) into (14) yields the potential equation

$$
\begin{aligned}
\nabla \cdot \mathbf{u} & =\nabla^{2} \Phi=\frac{g_{1}}{\rho_{s}}+\frac{g_{2}}{\rho_{v}}, \quad \mathbf{x} \in \mathrm{B}_{t}, \\
\Phi & =0, \quad \mathbf{x} \in \Gamma_{\text {int }}, \\
\mathbf{n}_{\Phi} \cdot \nabla \Phi & =0, \quad \mathbf{x} \in \Gamma_{\Phi} .
\end{aligned}
$$

In Eq. (15), $\mathbf{n}_{\Phi}$ denotes the norm vector of $\Gamma_{\Phi}$. Additionally, $\mathbf{u}=0$ in the domain outside of the biofilm $\mathrm{B}_{\mathrm{t}}$.

\subsection{Dimensionless Form of the Governing Equations}

Equations (1), (5), (10) and (15a) together with their corresponding boundary conditions compose the full mathematical model which describes the symbiotic biofilm system illustrated in Fig. 2.

To obtain the dimensionless form of the governing equations, we introduce the following dimensionless variables

$$
\begin{aligned}
& \mathbf{X}= \frac{\mathbf{x}}{H}, \quad T=\frac{t}{t_{\mathrm{d}}}, \quad \chi=\frac{t_{\mathrm{d}} \Phi}{H^{2}}, \quad S_{1}=\frac{s_{1}}{\overline{s_{1}}}, \\
& S_{2}=\frac{s_{2}}{\overline{s_{1}}}, \quad D_{2}^{*}=\frac{D_{2} t_{\mathrm{d}}}{H^{2}}, \quad \Theta_{11}^{2}=\frac{H^{2} \rho_{s} \mu_{1}}{Y_{s 1} D_{1} \overline{s_{1}}}, \quad \Theta_{12}^{2}=\frac{H^{2} \rho_{v} \mu_{2}}{Y_{v 1} D_{1} \overline{s_{1}}}, \\
& \Theta_{21}^{2}=\frac{\lambda \rho_{s} \mu_{1} t_{\mathrm{d}}}{\overline{s_{1}}}, \quad \Theta_{22}^{2}=\frac{\rho_{v} \mu_{2} t_{\mathrm{d}}}{\overline{s_{1}} Y_{v 2}}, \quad \Psi_{1}=\mu_{1} t_{\mathrm{d}}, \quad \Psi_{2}=\mu_{2} t_{\mathrm{d}},
\end{aligned}
$$




$$
K_{11}=\frac{k_{11}}{\overline{s_{1}}}, \quad K_{21}=\frac{k_{21}}{\overline{s_{1}}}, \quad K_{22}=\frac{k_{22}}{\overline{s_{1}}}, \quad K_{L}=\frac{k_{l}}{\overline{s_{1}}} .
$$

In Eq. (16), $H$ and $t_{\mathrm{d}}$ are characteristic length and time which are taken as the height of the computational domain and the time of a day respectively in this study.

Substituting Eq. (16) into Eqs. (1), (5), (10) and (15a) yields the dimensionless form of the governing equations. The detailed dimensionless governing equations are listed in Appendix A, and the corresponding numerical strategy for solving these dimensionless equations is presented in Appendices B and $\mathrm{C}$.

\section{Results}

We investigate the solution behaviors of the mathematical model in this section. An initially evenly mixed scenario has been investigated in Sect. 3.1. It turns out that the solution is spatially homogenized at a later time. To better understand the solution's homogenization property, we studied how different random initial conditions influence the solutions in Sect. 3.2. We also look into more realistic scenarios with an initial condition of one species embedded in another and investigate the role of symbiotic reactions in the generation of homogenized solutions (in Sects. 3.3 and 3.4).

Two-dimensional simulations are carried out with the modeling parameters listed in Table 1. For all numerical simulations, a $200 \times 200$ spatial mesh with four-node bilinear elements is applied and the time step size is taken as $\Delta t=\frac{1}{200}$ [day]. The TDG-FIC scheme (see Appendix B) with a 3rd order time accuracy is applied for solving the governing equations. In this study, we aim at investigating the reactive dynamics of the symbiotic biofilm system instead of a specific experimental scenario. We apply an initial condition for $S_{2}$ by assuming no lactic acid in the system as

$$
S_{2}(\mathbf{X}, t=0)=0, \quad \mathbf{X} \in \Omega^{*} .
$$

\subsection{Evenly Mixed Case}

A wave shape initial biofilm-fluid interface in the dimensionless domain is applied

$$
\Gamma_{\mathrm{int}}^{0}: \rightarrow \mathrm{Z}=0.1+0.05 \sin \left(4 \pi \mathrm{X}+\frac{\pi}{2}\right), \quad \mathbf{X} \in \Omega^{*} .
$$

We also consider that these two species of bacteria are initially evenly mixed as

$$
\vartheta_{1}(\mathbf{X}, t=0)=\vartheta_{2}(\mathbf{X}, t=0)=0.5, \quad \mathbf{X} \in \Omega^{*} \text {. }
$$

\subsubsection{Biomass Evolution and Distribution}

Simulation results of biomass distribution of both $S$. gordonii and Veillonella at early and late times are shown in Fig. 4. Since the numerical solutions are symmetric regarding the middle line $X=0.5$, only the half plane of a solution of each species of biomass 


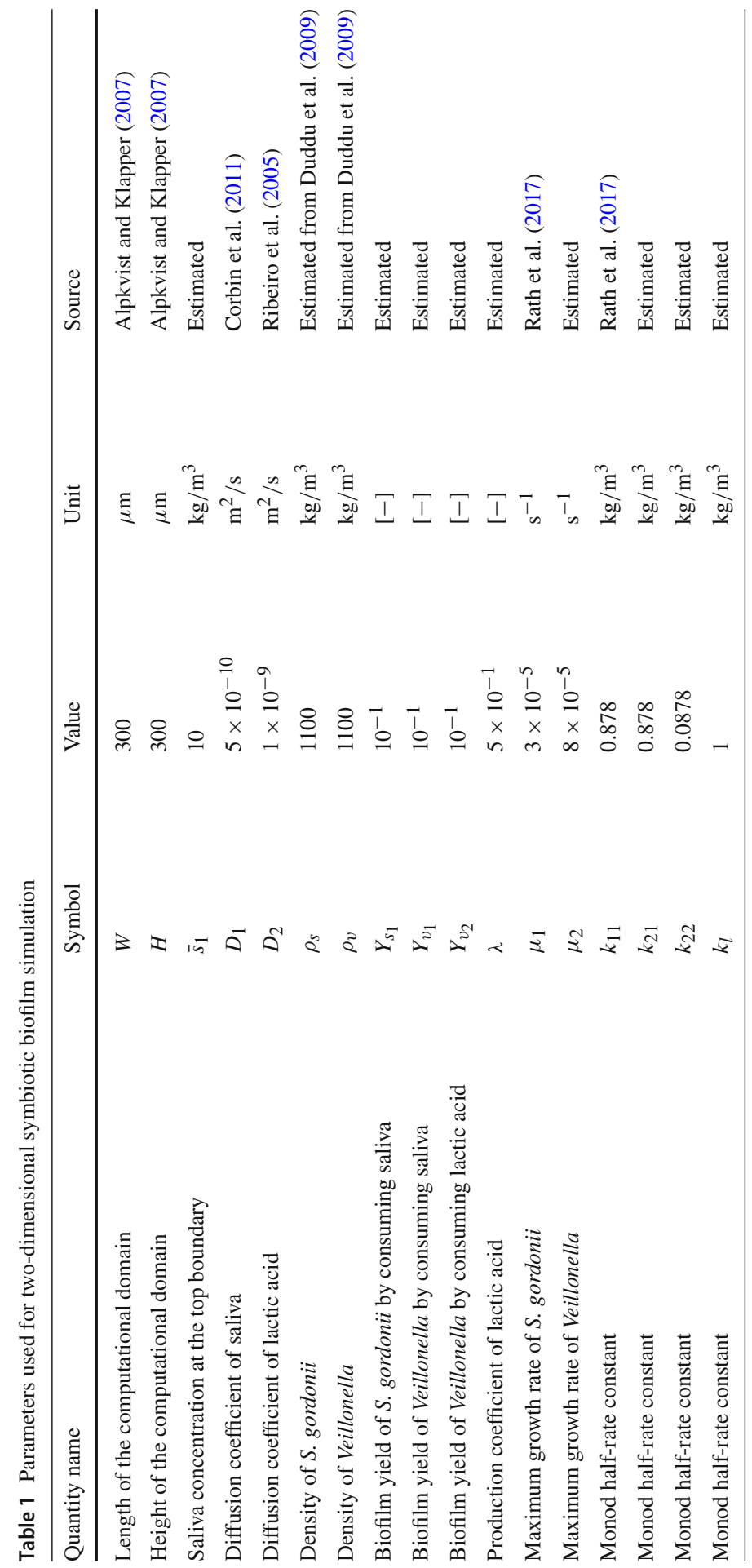




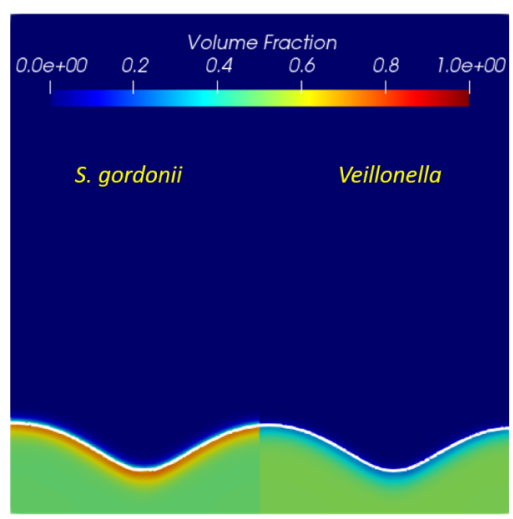

(a)

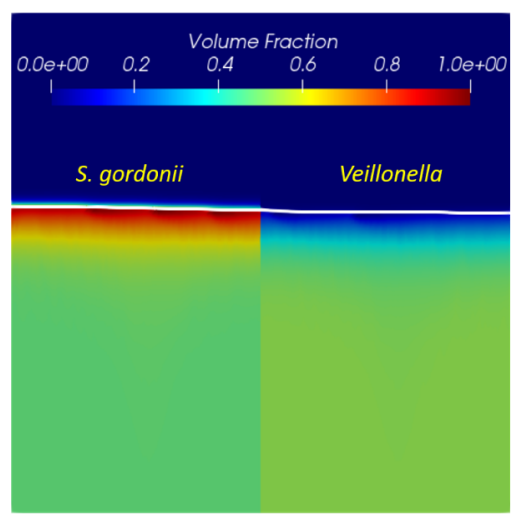

(c)

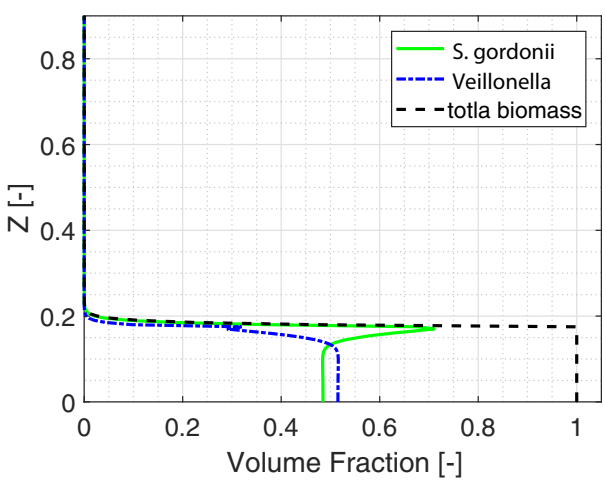

(b)

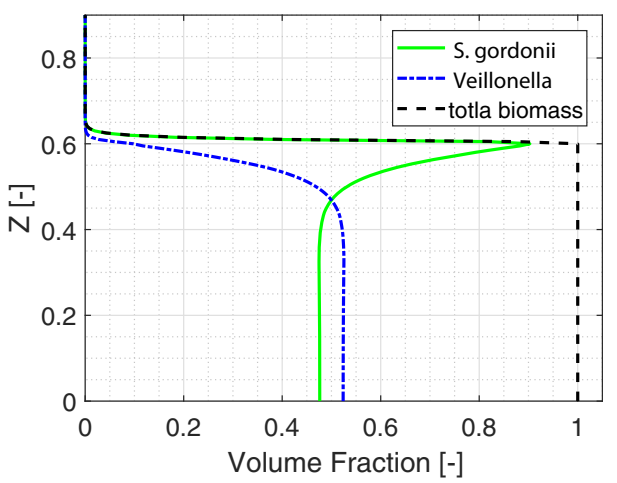

(d)

Fig. 4 Simulation results of the symbiotic biofilm system at different times (from top to bottom are at $t=9.6 \mathrm{~h}(T=0.4)$ and $t=48 \mathrm{~h}(T=2.0))$. Left column: Volume fraction distributions of $S$. gordonii and Veillonella; Right column: Volume fraction profiles of $S$. gordonii and Veillonella and total biomass in depth at $X=0.5$

is shown in the figure. The white curve in Fig. 4 represents the biofilm-fluid interface. The simulation results demonstrate that a flat biofilm pattern is generated after the growth of the biofilm for a certain time. This is due to the nutrients are not scarce in the system. However, we still can observe that the waved biofilm-fluid interface has quite an impact on the local distribution of the lactic acid (see later discussions).

Biomass volume fraction profiles of $S$. gordonii, Veillonella and total biomass at different times along $X=0.5$ are shown in the right column in Fig. 4. It is observed that $S$. gordonii moves up as a result of winning the competition for nutrients in saliva in the vicinity of the biofilm-fluid interface. Such a phenomenon has also been observed in experimental studies (Chalmers 2008) as shown in Fig. 5. In the figure, S. gordonii and Veillonella PK1910 are colored in green and blue respectively. We observe that the S. gordonii locates above the Veillonella in the areas within the red squares in Fig. 5. As a remark, we are not trying to reproduce the biofilm pattern shown in Fig. 5 in this 
Fig. 5 Spatial distribution of $S$. gordonii (in green) and Veillonella PK1910 (in blue) observed with confocal laser scanning microscopy in $2 \mathrm{~h}$ flowcell biofilms (Chalmers 2008)

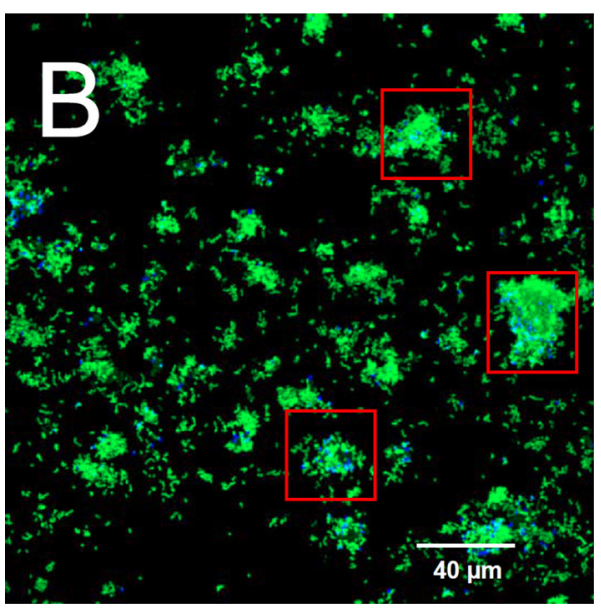

paper. The pattern of biofilms highly depends on nutrients conditions. A diluted saliva (Chalmers 2008) is used as a nutrient in the experiments corresponding to Fig. 5. We applied the calibrated model parameters corresponding to the growth of $S$. gordonii from our previous experimental study (Rath et al. 2017). The $S$. gordonii is cultured in the Tryptic Soy Broth medium instead of the diluted saliva. Under such a condition, we find the $S$. gordonii build up a biofilm of layering pattern (Rath et al. 2017; Kommerein et al. 2017). It has been observed that the Streptococcus sp. dominates when they are coaggregated with the Veillonella sp. Therefore, all numerical examples in this paper are set up with layered initial conditions.

The inhibition parameter $k_{l}$ has a significant influence on the growth of both species of bacteria. According to Eq. (3), the lactic acid does not prevent the growth of $S$. gordonii when $k_{l}$ is an infinitely large number. In this case, one would expect the $S$. gordonii grows at a large rate. As shown in Fig. 6, we observe a faster growth of both species of bacteria by using a larger value of $k_{l}$. As a remark, it is not surprised to see that the Veillonella sp. also grows faster associatively with the $S$. gordonii. This is due to the mutualistic relationship between these two species.

It should be noted that the simulation results correspond to an evenly mixed initial biomass volume condition. In nature, it is observed that S.gordonii usually attaches to a surface first and Veillonella attaches to Streptococcus afterwards. They form the so-called "corn cob" pattern (Kolenbrander et al. 2010) in vitro. Such a pattern demonstrates that the initial condition of either the geometry of the biofilm-fluid interface or the biomass distribution in such a symbiotic biofilm system is heterogeneous. To study such complex heterogeneity properties that are influenced by the initial conditions, an attachment process with considerations of bacteria receptors has to be considered. The attachment process normally happens at an individual bacterium scale and can hardly be considered only with a continuum model. To capture the attachment process, modeling the whole biofilm system with a multi-scale model will be promising. However, this is beyond the scope of this paper. 


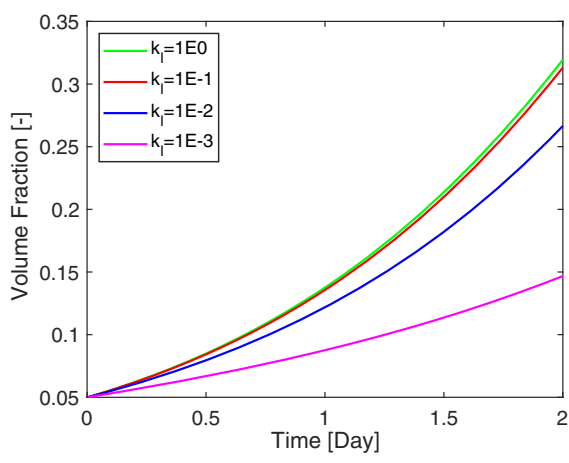

(a) Volume fraction evolution profiles of Streptococcus sp. with different inhibition parameter $k_{l}$.

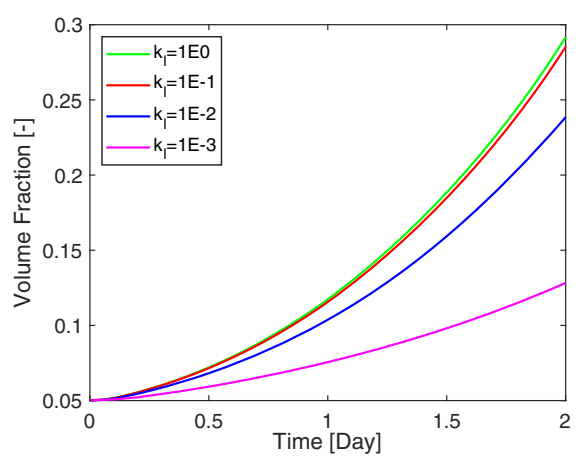

(b) Volume fraction evolution profiles of Veillonella sp. with different inhibition parameter $k_{l}$.

Fig. 6 Sensitivity analysis of the inhibition parameter $k_{l}$ on the growth of bacteria

\subsubsection{Lactic Acid}

Distribution of the lactic acid in terms of its dimensionless concentration $S_{2}$ at different times is presented in Fig. 7. The lactic acid is produced by the S. gordonii and consumed by the Veillonella. The acid is transported diffusively and accumulates in the fluid domain. It is observed that a higher concentration of acid accumulates above the biofilm-fluid interface, especially, after the $S$. gordonii becomes dominant at the top layer of the biofilm. Since there is more $S$. gordonii at the valley of the biofilm surface at $T=1.0$ and $T=1.6$, two punctiform sources of the acid at the biofilm valley are observed. This is why we observe the maximum acid concentration appears in the middle of the computational domain, and the acid diffuses to the top boundary. The Veillonella wins the competition at the lower part of the biofilm (see biomass profiles in Fig. 4) where the lactic acid is largely consumed by the Veillonella. What should be noted is that the distribution of the lactic acid is also influenced by the biofilm patterns and the generation of biofilm patterns might be influenced by the distribution of the lactic acid as well.

Figure 8 shows profiles of the lactic acid at $X=0.5$ at different times. For each plot, the concentration of the lactic acid increases from the bottom of the biofilm to the top of it and decreases slightly afterwards in the bulk fluid. The profiles demonstrate that the location of the maximum concentration moves together with the biofilm interface. The profiles also show that the lactic acid accumulates in the bulk fluid domain over time. Comparing the two profiles of $t=1.6 \operatorname{day}(T=1.6)$ and $t=2.0 \operatorname{day}(T=2.0)$, the difference of the acid concentrations at the bottom of the biofilm is rather small. It seems the solution reaches a steady state locally there.

Since we apply a no-flow boundary to model the acid transport in a batch system. The acid accumulates in the fluid domain over time. One can also apply a Dirichlet boundary for $s_{2}$. By assuming the acid is removed immediately at the top of the 


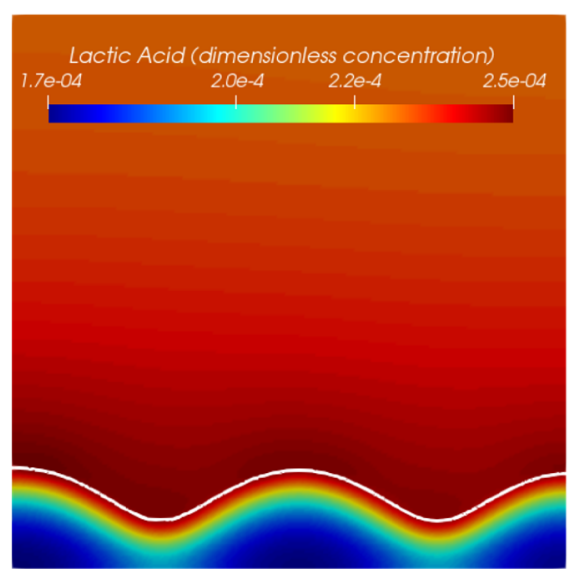

(a) $T=0.4$

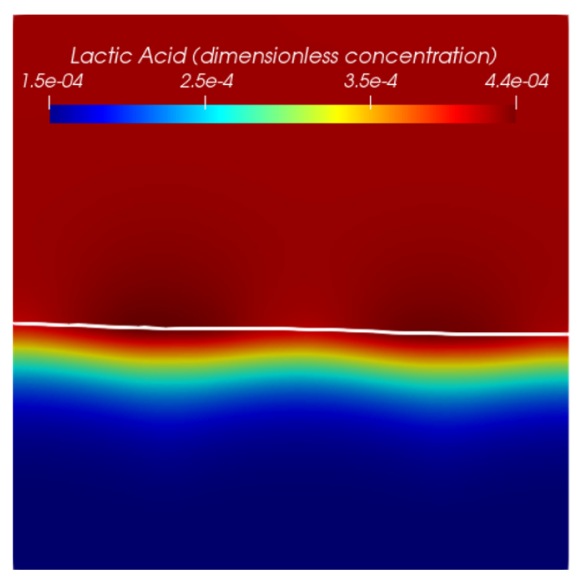

(c) $T=1.6$

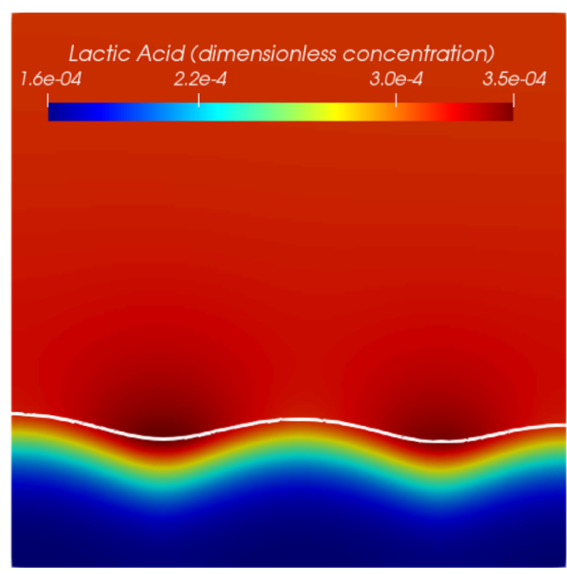

(b) $T=1.0$

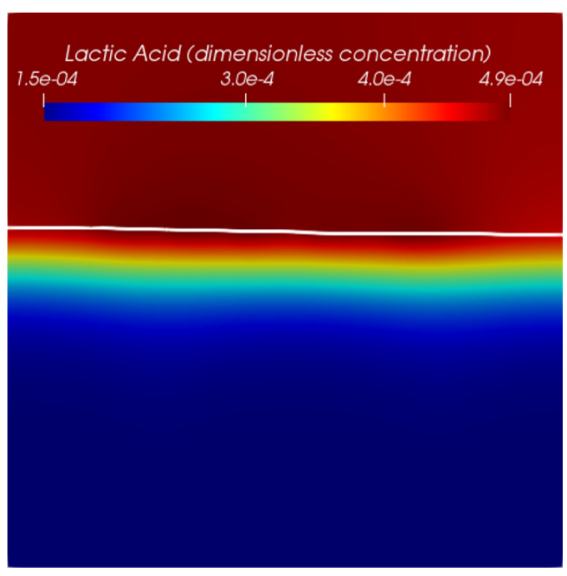

(d) $T=2.0$

Fig. 7 Distribution of lactic acid of the symbiotic biofilm system at different times $(T=0.4,1.0,1.6,2.0)$. The white curve in the figures denotes the biofilm-fluid interface

computation domain, Eq. (13) can be replaced by

$$
s_{2}=0, \quad \mathbf{x} \in \Gamma_{\mathrm{ht}} .
$$

As shown in Fig. 9, the lactic acid profiles are different than the profiles shown in Fig. 8 in the fluid domain. We further compare the solutions of the lactic acid by using different boundary conditions in Fig. 10. It turns out that different boundary conditions do not affect the acid profiles much in the biofilm.

The lactic acid plays a twofold role in biofilm growth. On the one hand, it is the carbon source for Veillonella. On the other hand, the acid inhibits the growth of Streptococcus. In this study, we apply a relatively large value of $k_{l}$ (see Table 1), which 
Fig. 8 Dimensionless concentration profiles of lactic acid at $X=0.5$ at different times $(T=0.4,1.0,1.6,2.0)$ with a no-flow boundary condition

Fig. 9 Dimensionless concentration profiles of lactic acid at $X=0.5$ at different times ( $T=0.4,1.0,1.6,2.0)$ with a Dirichlet boundary condition
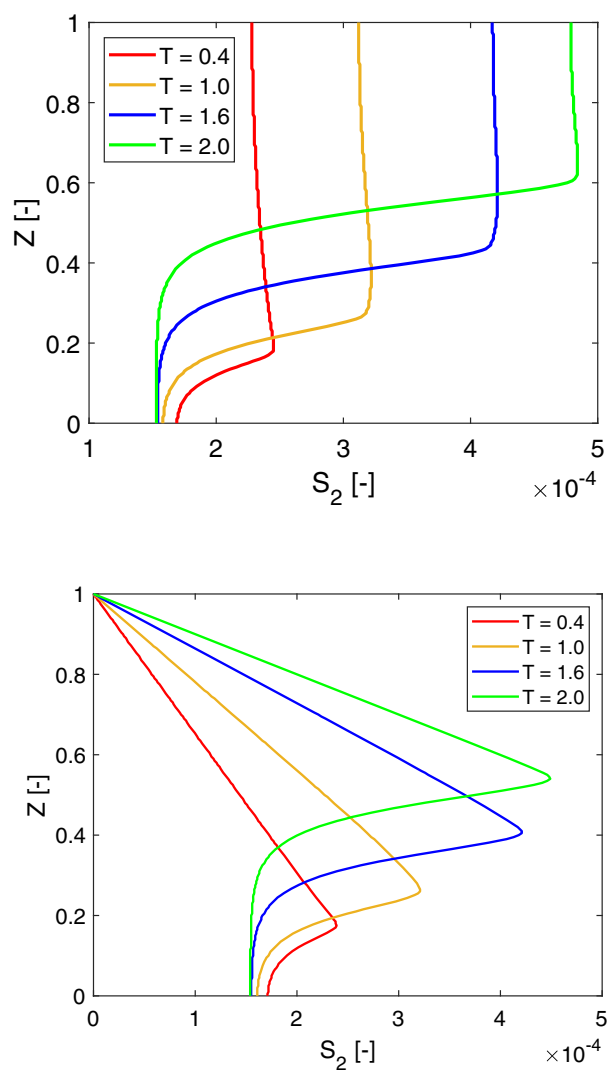

reduces the second effect. Since the acid does not accumulate in the bulk fluid when the Dirichlet boundary is applied, its concentration is relatively smaller, especially at a later time (e.g., the peak value of $S_{2}$ at $T=2.0$ ). Therefore, the biofilm grows slower. The dimensionless thickness of the biofilm reduces $3 \%$ at $T=1.6$ by using the Dirichlet boundary. At $T=2.0$, the reduction is increased to $6 \%$. This study aims to investigate the solution behaviors, the boundary condition of the acid will not affect our investigation results.

The simulation results of this $2 \mathrm{D}$ example shed a light on the properties of the multi-dimensional numerical solutions of the presented biofilm model. First, heterogeneous biomass distribution is obtained with this model even though a fully mixed initial condition is applied. On the other hand, the biomass is homogenized locally in the biofilm. Second, the biofilm-fluid interface is flatted in time. However, the non-flat interface has an influence on the lactic acid distribution. Based on those observations, we ask ourselves: (1) Will the local homogenization process always happen in this model regardless of the initial biomass distribution? If so, will the initial distribution influence the homogenization process? (2) How will a heterogeneous initial biomass distribution influence the morphology of the biofilm interface as well as the components (including the biomass and the lactic acid) distributions during the growth? 
Fig. 10 Comparison of lactic acid profiles at $X=0.5$ with different boundary conditions at $T=0.4,1.6$ and 2.0

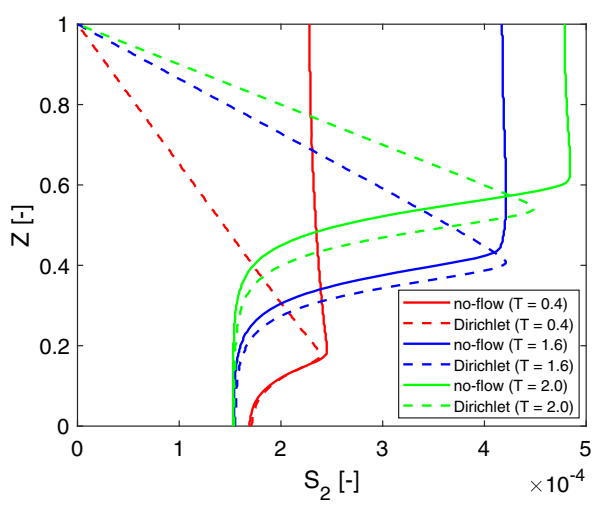

\subsection{Sensitivity to Initial Biomass Distribution}

In order to answer these questions raised in the previous section, we study the sensitivity of the presented mathematical model to different types of initial biomass distributions. As a matter of fact, the initial distribution of different species of bacteria in a biofilm can be never evenly mixed in nature. As shown in Fig. 11, we consider three cases of the random initial distribution of biomass. Random fields are generated by using given different correlation lengths. The correlation length measures the distance of two correlated points (e.g., the points with correlated material properties or physical quantities). Thus, the correlation length is often seen as a measure of the roughness of surfaces. In this study, different correlation lengths of $2 \Delta x, 4 \Delta x$, and $8 \Delta x$ are applied in each case, in which $\Delta x=\frac{1}{200}$ denotes the length of a spatial element in the horizontal direction. As a remark, the total initial mass (or volume) of each species of bacteria is the same. To simplify the problem, the initial biofilm-fluid interface is modeled as a flat line, and a constant initial biofilm thickness of $60 \mu \mathrm{m}$ $(Z=0.2)$ is assumed. Following our previous numerical setup, we assume that there is no lactic acid in the system initially.

As shown in Fig. 12, the Veillonella patches are stretched and thus generating plume-like structures during the biofilm formation in all three cases. This indicates that processes happen mainly in the vertical direction, and the horizontal movement is not obvious. By increasing the correlation length of the initial biomass distribution, the size of the plumes increases. This results in that the biomass volume fraction profiles along a vertical line, for instance at $x=150 \mu \mathrm{m}(X=0.5)$ as shown in Fig. 13, get less fluctuated with a larger correlation length. However, this does not mean that with a larger correlation length, the biomass homogenizes faster. In order to study how different biomass initial distributions influence the homogenization time, we compare the numerical solutions corresponding to the random initial distributions to the solution with an evenly mixed initial biomass distribution. Since the simulation involves moving domains, measuring the homogenization process is nontrivial. We calculate the difference of the volume fractions of the Veillonella between the evenly 


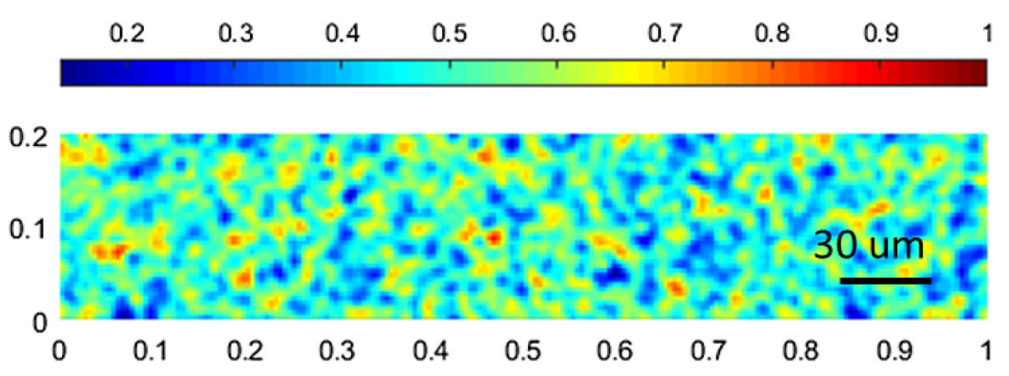

(a) correlation length: $2 \Delta x$
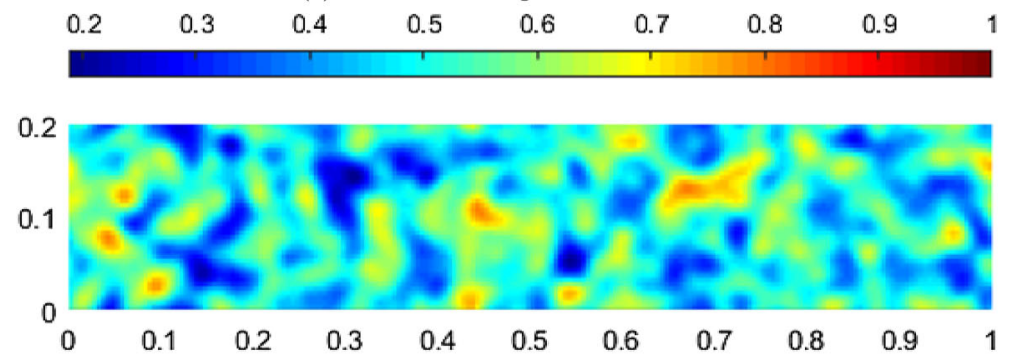

(b) correlation length: $4 \Delta x$
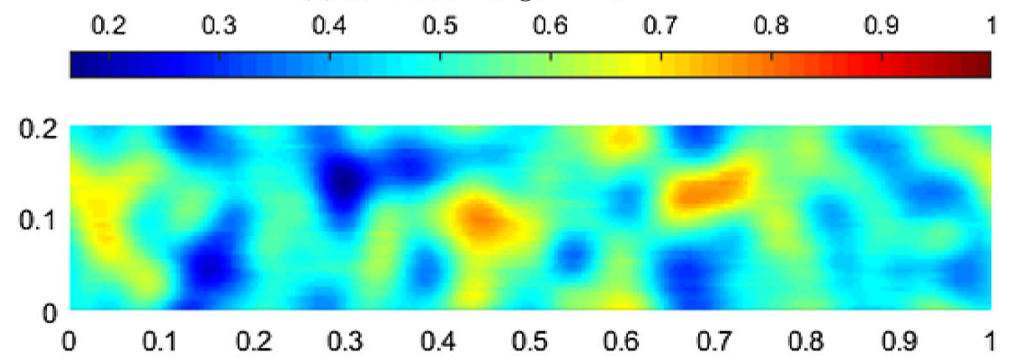

(c) correlation length: $8 \Delta x$

Fig. 11 Initial random volume fraction distribution of Veillonella with different correlation lengths

mixed and randomly distributed cases to measure the homogenization process

$$
M_{\text {diff }, i}=\frac{1}{N}\left\|\left.\vartheta_{2}\right|_{\text {mixed }}-\left.\boldsymbol{\vartheta}_{2}\right|_{i}\right\|,
$$

where $\boldsymbol{\vartheta}_{2}$ is the solution vector of the volume fraction of Veillonella, $N$ is the number of the unknowns in the vector and indexes "mixed" refers to the evenly mixed initial condition. The index $i=1,2,3$ refers to scenarios with different correlation lengths of $2 \Delta x, 4 \Delta x$ and $8 \Delta x$. Since the evenly mixed case always presents a locally homogenized solution which produces a comparable biofilm thickness evolution as the other cases at an early time, a smaller $M_{\text {diff }}$ corresponds to a solution which is more homogenized. 


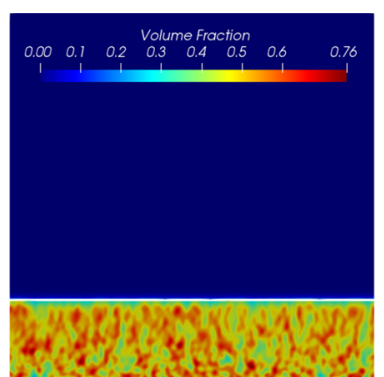

(a)

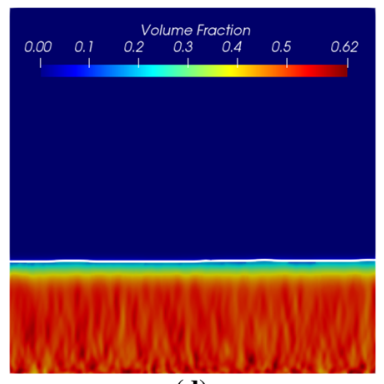

(d)

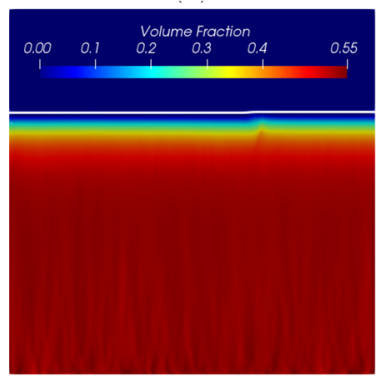

(g)

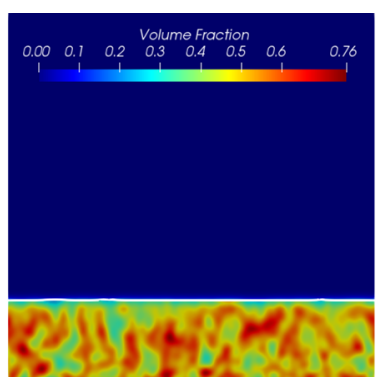

(b)

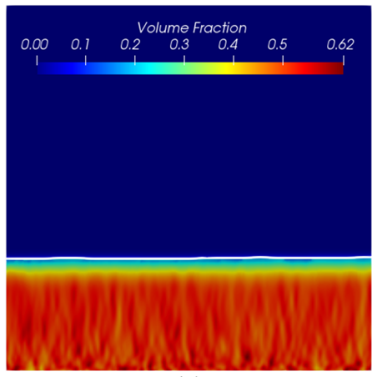

(e)

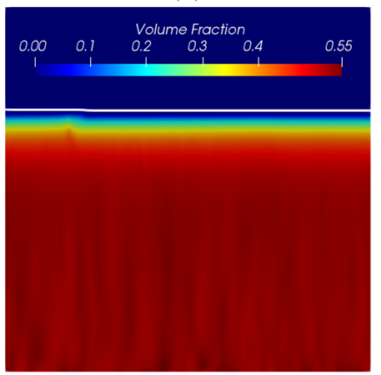

(h)

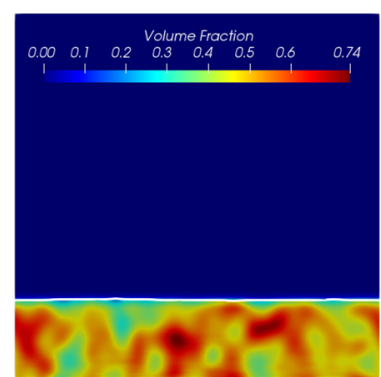

(c)

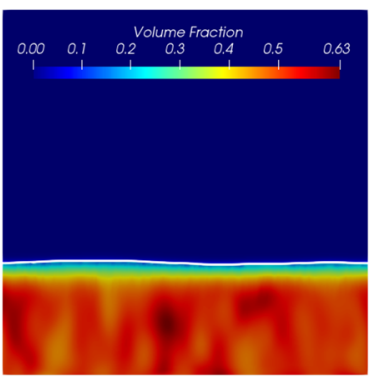

(f)

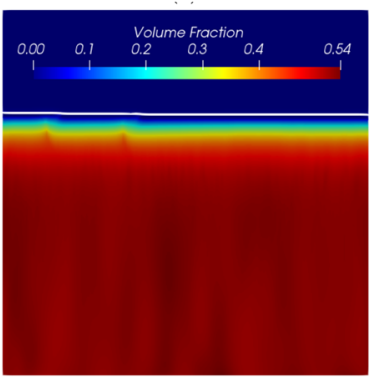

(i)

Fig. 12 Volume fractions of Veillonella at different times corresponding to different initial distribution with various corrlation lengths. Figure from left to right refer to simulation results corresponding to different initial correlation lengths of $2 \Delta x, 4 \Delta x$ and $8 \Delta x$. Figure from top to bottom refer to simulation results at different times of $t=0.1$ day $(T=0.1), t=0.5$ day $(T=0.5)$ and $t=1.5$ day $(T=1.5)$

The evolution of $M_{\text {diff }}$ corresponding to these three scenarios is illustrated in Fig. 14. The results demonstrate that the numerical solutions are homogenized in all these three cases as soon as the simulation starts. The case corresponding to a smaller correlation length requires less time to reach the state of a given value of $M_{\text {diff }}$ at the early time. In other words, the system homogenizes faster when the correlation length is smaller. However, as mentioned earlier, more fluctuated vertical biomass profiles are observed corresponding to the scenarios with larger correlation length. Moreover, $M_{\text {diff }}$ increases at a later time (as shown in Fig. 14). This is due to even though the initial biomass in the homogeneous case is the same as these three heterogeneous cases, the distribution of the lactic acid is different from case to case which results 
Fig. 13 Volume fraction profiles of Veillonella at $X=0.5$ at $t=0.1$ day $(T=0.1$; early time), $t=0.5$ day ( $T=0.5$; middle time) and $t=1.5$ day ( $T=1.5$; late time)

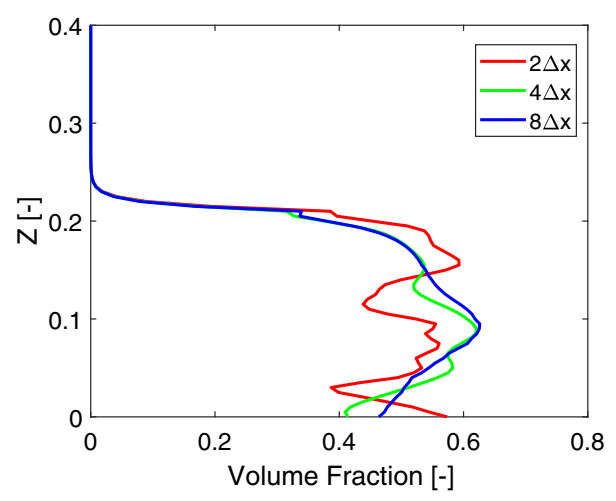

(a) $t=0.1$ day

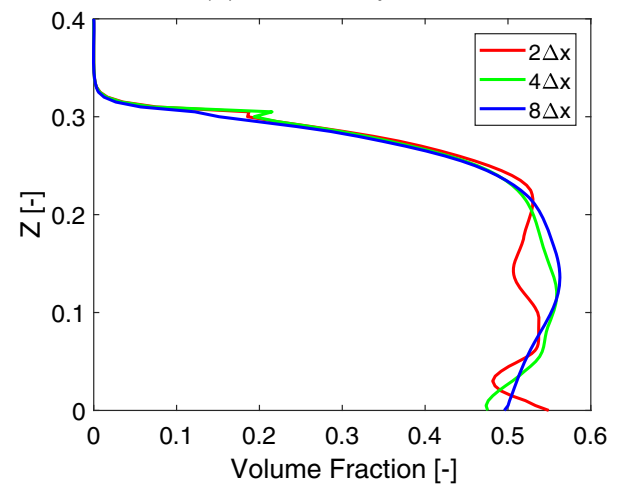

(b) $t=0.5$ day

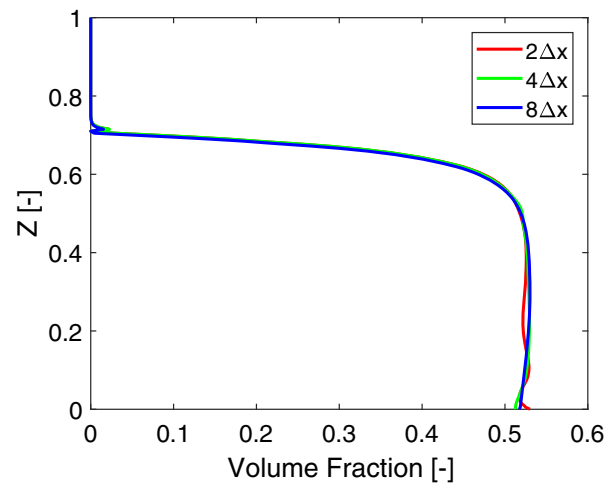

(c) $t=1.5$ day

in different biomass production in the heterogeneous cases than in the homogeneous case. Such a difference may accumulate over time and leads to an increase in the measured mass difference $M_{\text {diff }}$ at late time. In a word, the presented heterogeneous system will not converge to the homogeneous system where the biomass is initially evenly mixed. 
Fig. 14 Homogenization times of three scenarios corresponding to initial biomass distribution with different correlation lengths $2 \Delta x, 4 \Delta x$ and $8 \Delta x$

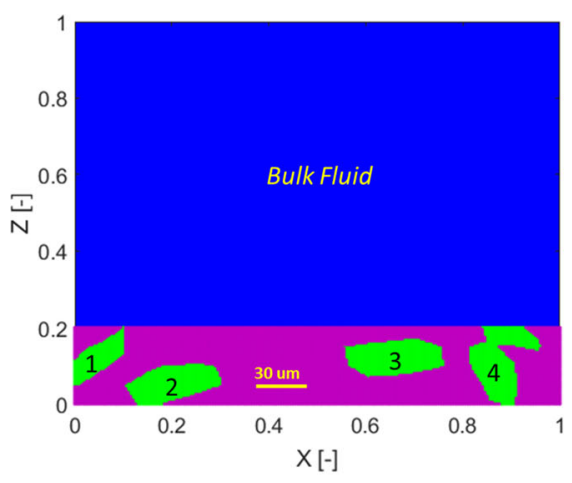

(a) case a)
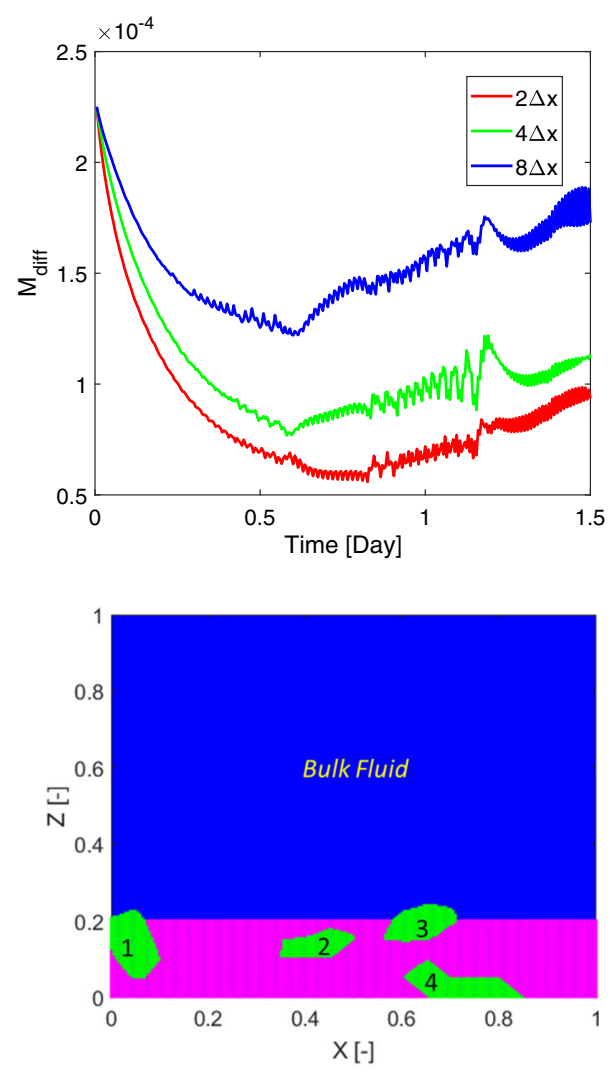

(b) case b)

Fig. 15 Two scenarios of initial biomass distribution (blue:bulk fluid; purple: S. gordonii; green: Veillonella)

\subsection{Initial Biomass Distribution with Patch-shape Veillonella}

One could also argue that a random initial distribution of biomass is not realistic. It is more often found in mature biofilm systems that the Veillonella group bacteria are embedded in Streptococcus group bacteria (Chalmers et al. 2008; Kommerein et al. 2017). Therefore, we carry out studies with two different initial biomass distribution scenarios as shown in Fig. 15. The irregular geometry of the Veillonella clusters, numbered from 1 to 4 (from left to right), are generated by using an implicit superquadric function which is defined by an implicit equation with random parameters (Wellmann and Wriggers 2012) and is rotated by a random angle. In case (a), the initial biofilm-fluid interface is flat, while a non-flat initial interface is assigned in case (b).

Simulation results of these two cases with different initial biomass distributions are shown in Figs. 16 and 17. The simulation results demonstrate that the locally homogenization occurs at a late time (at 1.0 day). Some Veillonella patches are still observable even at $T=1.0$. One may expect that the patches will be homogenized if we run the simulation long enough. Also, a non-flat biofilm-fluid interface is generated 


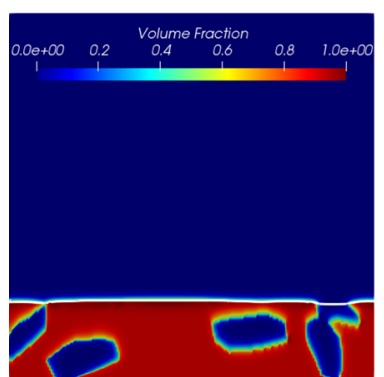

(a)

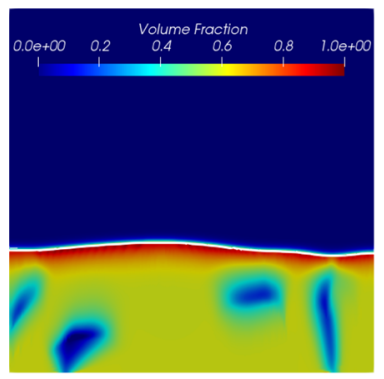

(d)

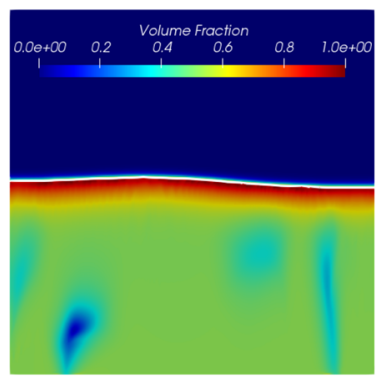

(g)

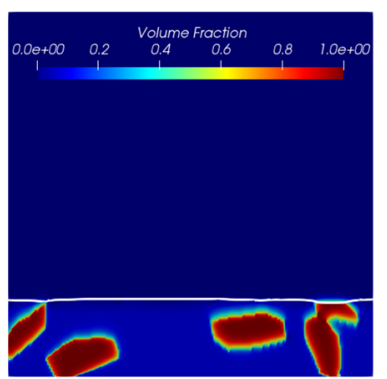

(b)

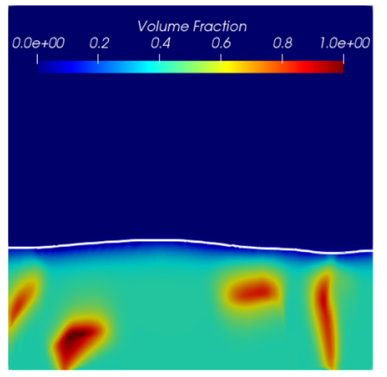

(e)

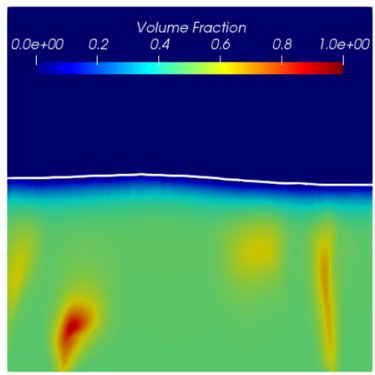

(h)

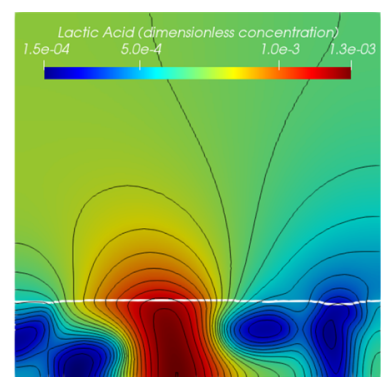

(c)

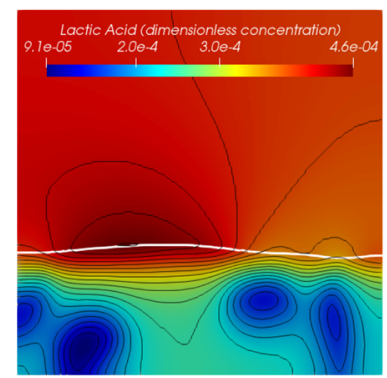

(f)

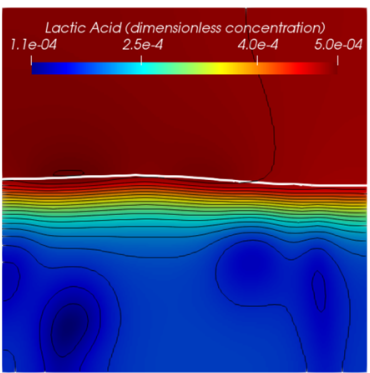

(i)

Fig. 16 Simulation results of the symbiotic biofilm system at different times (corresponding to the case in Fig. 15a). The white curve denotes the biofilm-fluid interface. From top to bottom are at $t=0.05$ day $(T=0.05), t=0.5$ day $(T=0.5)$ and $t=1.0$ day $(T=1.0)$. Left column: Volume fraction distributions of S. gordonii; Middle colume: Volume fraction distributions of Veillonella; Right column: Dimensionless concentration of lactic acid (black curves denote isolines of $S_{2}$ )

during the growth even if the interface is flat initially (as shown in Fig. 16). This is due to the heterogeneous reactions, which result in a heterogeneous biofilm growth velocity field. With the homogenization of the solution, the biofilm interface gets flatted again

Contours of the volume fraction of Veillonella $\vartheta_{2}=0.6$ of each case at $t=0$ and at $t=1.0$ day are plotted in Fig. 18. In Fig. 18a, the second Veillonella cluster (numbered in Fig. 15) seems to be rotated anticlockwise. However, the third cluster moves up together with the growth of the biofilm. The forth one is simply stretched and shrunken. In case (b), as shown in Fig. 18b, we observe an interesting feature of the 


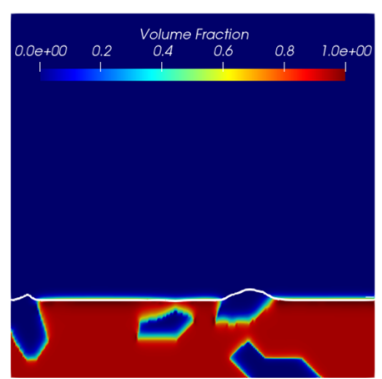

(a)

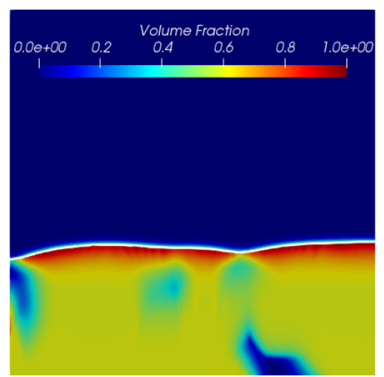

(d)

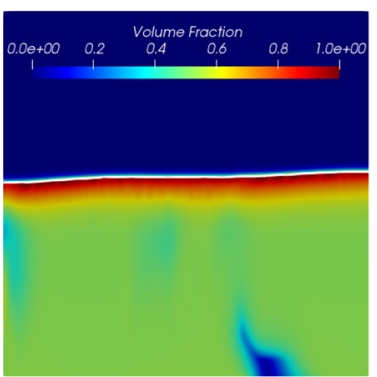

(g)

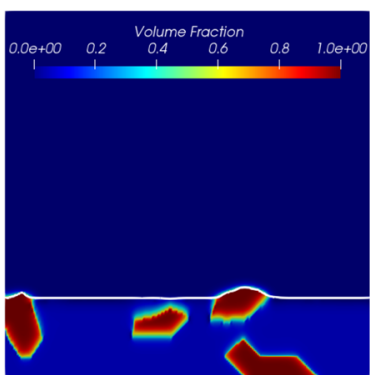

(b)

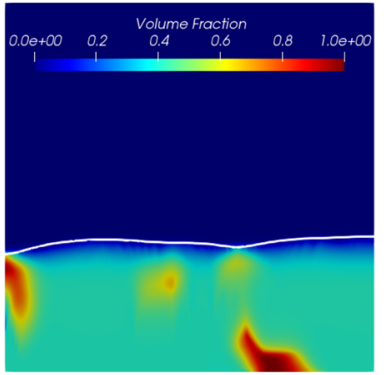

(e)

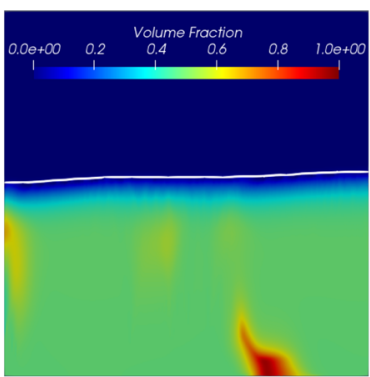

(h)

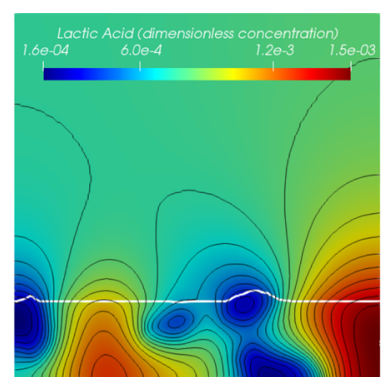

(c)

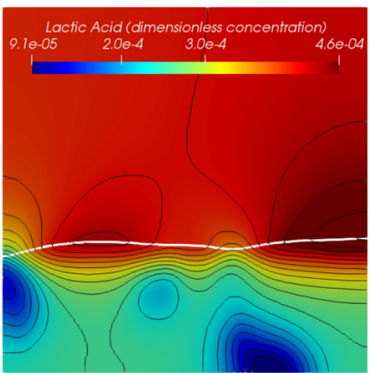

(f)

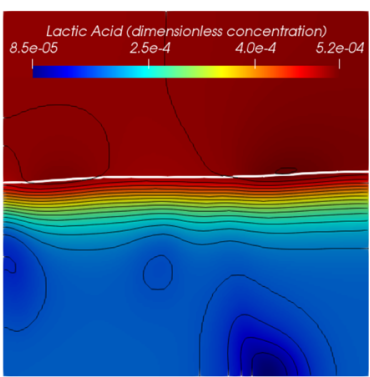

(i)

Fig. 17 Simulation results of the symbiotic biofilm system at different times (corresponding to the case in Fig. 15b). The white curve denotes the biofilm-fluid interface. From top to bottom are at $t=0.05$ day $(T=0.05), t=0.5$ day $(T=0.5)$ and $t=1.0$ day $(T=1.0)$. Left column: Volume fraction distributions of S. gordonii; Middle colume: Volume fraction distributions of Veillonella; Right column: Dimensionless concentration of lactic acid (black curves denote isolines of $S_{2}$ )

numerical solution that the third and the forth Veillonella clusters cannot be captured by the contour line $\vartheta_{2}=0.6$ at 1.0 day. This demonstrates that those two clusters are homogenized faster than the other two clusters. The simulation results suggest that the homogenization time varies spatially which might be induced by the heterogeneous reactions. 


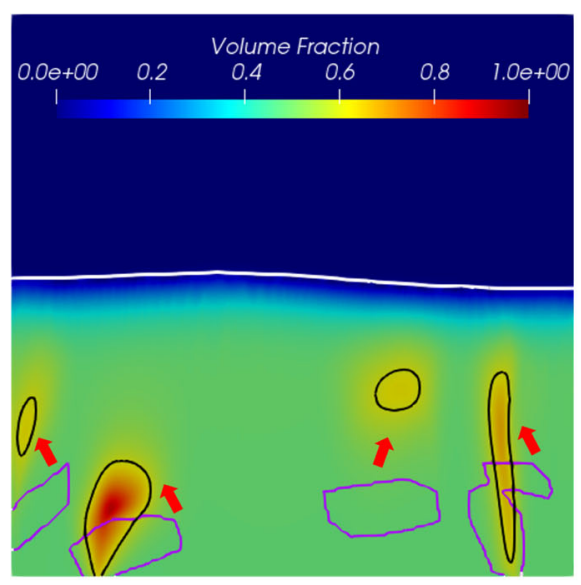

(a) case a)

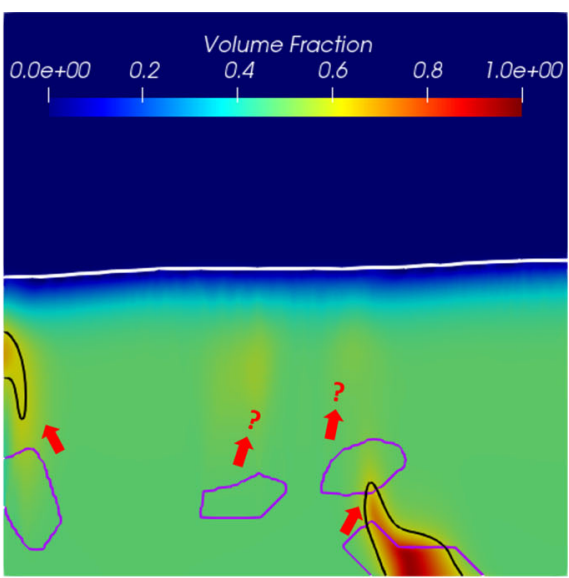

(b) case b)

Fig. 18 Movement of Veillonella clusters over time. The purper curve and the black curve denote contour lines of volume fraction of Veillonella $\vartheta_{2}=0.6$ at $t=0$ day $(T=0)$ and $t=1.0$ day $(T=1.0)$ respectively

\subsection{The Role of the Symbiotic Reactions}

Following the discussions in the previous section, the presented reaction model may be one of the main reasons that cause local biomass homogenization. To understand the role of the reaction terms in the mathematical model, we compare the simulation results presented in Fig. $18 \mathrm{~b}$ to the results by using a model without symbiotic reactions. The same model setup, as the case shown in Fig. 18b, has been applied in this study. We simplify the symbiotic model (SM) presented in Sect. 2 by applying:

$$
\begin{aligned}
& g_{1}=\vartheta_{1} \rho_{s} \mu_{1} \frac{s_{1}}{k_{11}+s_{1}}, \\
& g_{2}=\vartheta_{2} \rho_{v} \mu_{2} \frac{s_{1}}{k_{21}+s_{1}},
\end{aligned}
$$

and

$$
r_{22}=0
$$

The model reduces to a competitive model (CM) in which the growth of both species of bacteria is limited only by the nutrient (saliva). In this case, the lactic acid does not play a role in biofilm growth, and it accumulates in space. One would expect that by applying CM, the biofilm grows faster than using SM due to the absent limitation of the lactic acid. Meanwhile, the species which has a larger maximum growth rate becomes dominant during the growth. As shown in Fig. 19, the simulation results of the Veillonella sp. by using CM meet the above expectations. The Veillonella sp. becomes dominating during the growth and wins the competition. The simulation results of the CM (as shown in Fig. 19) do not homogenized as in the SM (see Fig. 18b). This 


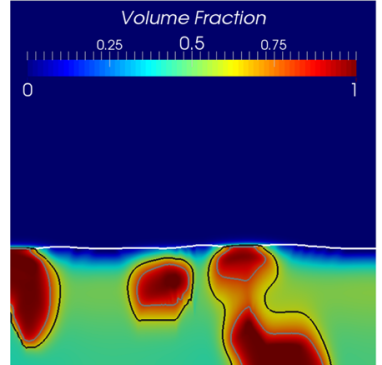

(a) $t=0.25$ day

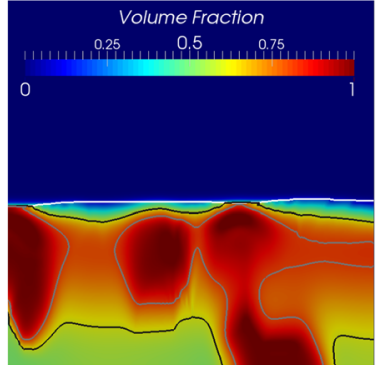

(b) $t=0.5$ day

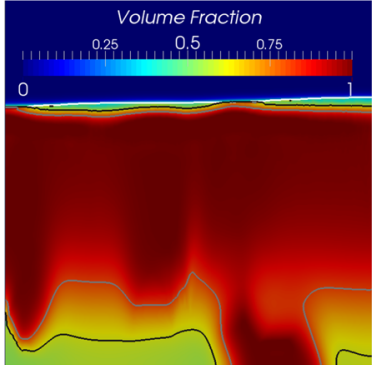

(c) $t=1.0$ day

Fig. 19 Simulation results of volume fractions of Veillonella sp. at different times by using the competitive model. The white curve denotes the biofilm-fluid interface. The black and grey curves denote contour lines of volume fraction of Veillonella $\vartheta_{2}=0.6$ and $\vartheta_{2}=0.8$ respectively

demonstrates that the symbiotic interaction is one of the main reasons that lead to the homogenized solutions.

\section{Summary and Discussion}

\subsection{Summary}

We have presented a new mathematical model for modeling the growth of symbiotic biofilms in this paper. An intermediate product, namely the lactic acid produced by the Streptococcus sp., has been explicitly modeled. We found that the volume fractions of different species of bacteria homogenize at a later time. To understand the solution behaviors, we studied how the initial biomass distribution influences the homogenization process. Random distributions of the biomass volume fractions with different correlation lengths were taken as initial conditions. We compared the simulation results of the case where the bacteria were initially evenly mixed. As would be expected, the biomass homogenized faster associating with an initial state with a smaller correlation length. However, the random distribution cases do not converge to the same concentration distribution as the evenly mixed one, although the same initial mass and morphology of the biofilm surface (flat surface) have been assigned.

For a better understanding of the role of the initial distributions, we investigated scenarios with patch-shape biomass. The simulation results demonstrate that the local growth field can lead to changes in the morphology of the biofilm surface and different bacteria patches' movements. Moreover, we also observe various homogenization speeds that are induced by the heterogeneity of the symbiotic reactions. To understand the role of the reactions in the homogenization process, we simplified the model by switching off the symbiotic reactions, resulting in a competitive model. The competitive model's numerical solution does not get homogenized, which demonstrates that the symbiotic reaction is one of the main reasons for spatial homogenizations. 


\subsection{Discussion}

It is well-known in microbiology that bacterial biofilms' biological behaviors are a lot more complicated than a mere competition of "food" in nature. Many efforts have been put in the past decades to understand how different bacteria species interact in a biofilm. The symbiotic relationship between two species of bacteria is one of the most interesting phenomena in microbiology systems.

Mathematical modeling has been proved to be a powerful tool for understanding biofilm's biological behaviors in the past years (Mattei et al. 2017). Tens or even hundreds of mathematical models have been developed for modeling the bacterial biofilm systems since the 1980s (Rittmann and McCarty 1980). Nowadays, biofilm models are getting more comprehensive than earlier ones due to a deeper understanding of the biological mechanisms. Based on the previous pioneer continuum biofilm models (Alpkvist and Klapper 2007; Klapper and Dockery 2002), we studied a dual-species biofilm system's symbiotic behavior by using mathematical modeling.

To the best of the authors' knowledge, this study provides the first comprehensive investigation of the dynamics of a biofilm's symbiotic reactions using a continuum mathematical model. We modeled the biofilm growth as an advective movement of a reactive potential flow in this study. The model has the advantage of capturing a sharp biofilm interface without introducing additional parameters; however, it suffers limitations on modeling merge of different colonies (see Discussion in Sect. 2). To overcome this drawback, one can improve the model by considering the fluid phase explicitly and thus lead to the mixture models (Zhang et al. 2008a). Alternatively, one can model the biofilm growth as a diffusion process (Ghasemi et al. 2018; Rahman et al. 2015), and apply the reaction models presented in this paper directly.

Instead of modeling the colonies' merge, we are more interested in the reaction model developed for the symbiotic biofilm systems. Even though the simulation results demonstrate that the model can represent the species of bacteria's symbiotic behaviors, we found that the reactive model can lead to homogenized solutions. The presented model may fail to predict the biological behavior of the symbiotic biofilms after a certain time when the numerical solution becomes over-homogenized. Therefore, it is necessary to investigate what factors influence the homogenization speed in the model. It turned out that the speed of the homogenization depends on the initial biomass distribution.

Individual bacteria are never resolved in a continuum biofilm model. This might be another potential reason which leads to the over-mixing of the biomass. However, the discretized element based biofilm models can also result in considerable internal mixing, especially for multi-species problems (Tang and Valocchi 2013). It would be interesting to compare the mixing behavior occurred in different continuum and discretized element based biofilm models in future studies. As a matter of fact, there are still many open questions on modeling multi-species biofilm problems. A third possible reason that results in the unwanted homogenized solution is missing important bioprocesses in the model. There could be specific quorum sensing (QS) mechanisms that prevent the mixing process in nature. Egland et al. presented experimental evidence of signaling between S. gordonii and V. atypica (Egland et al. 2004). The diffusive 
short distance signaling induced by the quorum sensing (QS) might be one of the key reasons that lead to the "corn cob" pattern of the biofilm system. Mathematical modeling the QS in biofilm systems has already attracted much attention in recent years (Emerenini et al. 2015; Zhao and Wang 2017; Ghasemi et al. 2018). However, many more detailed QS mechanisms are still unclear (Egland et al. 2004) and may result in many uncertainties in the model.

Acknowledgements The authors would like to thank the reviewer for his/her constructive comments and efforts towards improving our manuscript. Thanks also go to Dr. Henryke Rath, Dr. Nadine Kommerein and Mrs. Natascha Brandhorst for many helpful discussions. Dianlei Feng would also like to thank the German Research Council (DFG) under grant No. FE 1962/1-1 (426819984).

Funding Open Access funding enabled and organized by Projekt DEAL. DFG Funding Instrument "Temporary Positions for Principal Investigators".

\section{Declarations}

Conflict of interest The authors declare that they have no conflict of interest.

Open Access This article is licensed under a Creative Commons Attribution 4.0 International License, which permits use, sharing, adaptation, distribution and reproduction in any medium or format, as long as you give appropriate credit to the original author(s) and the source, provide a link to the Creative Commons licence, and indicate if changes were made. The images or other third party material in this article are included in the article's Creative Commons licence, unless indicated otherwise in a credit line to the material. If material is not included in the article's Creative Commons licence and your intended use is not permitted by statutory regulation or exceeds the permitted use, you will need to obtain permission directly from the copyright holder. To view a copy of this licence, visit http://creativecommons.org/licenses/by/4.0/.

\section{A Dimensioless Form of Governing Equations}

\section{Mass balance for saliva}

$-\nabla^{2} S_{1}=-\Theta_{11}^{2} \vartheta_{1} \frac{S_{1} K_{L}}{\left(K_{11}+S_{1}\right)\left(K_{L}+S_{2}\right)}-\Theta_{12}^{2} \vartheta_{2} \frac{S_{1} S_{2}}{\left(K_{21}+S_{1}\right)\left(K_{22}+S_{2}\right)}, \quad \mathbf{X} \in \Omega^{*}$.

\section{Mass balance for lactic acid}

$$
\begin{aligned}
& \frac{\partial S_{2}}{\partial T}+\nabla \chi \cdot \nabla S_{2}-D_{2}^{*} \nabla^{2} S_{2} \\
& \quad=\Theta_{21}^{2} \vartheta_{1} \frac{S_{1} K_{L}}{\left(K_{11}+S_{1}\right)\left(K_{L}+S_{2}\right)}-\Theta_{22}^{2} \vartheta_{2} \frac{S_{1} S_{2}}{\left(K_{21}+S_{1}\right)\left(K_{22}+S_{2}\right)}- \\
& S_{1} S_{2}\left[\frac{\vartheta_{1} \Psi_{1} K_{L}}{\left(K_{11}+S_{1}\right)\left(K_{L}+S_{2}\right)}+\frac{\vartheta_{2} \Psi_{2} S_{2}}{\left(K_{21}+S_{1}\right)\left(K_{22}+S_{2}\right)}\right], \quad \mathbf{X} \in \Omega^{*} .
\end{aligned}
$$


Mass balance for S. gordonii

$$
\begin{aligned}
& \frac{\partial \vartheta_{1}}{\partial T}+\nabla \chi \cdot \nabla \vartheta_{1} \\
& \quad=\vartheta_{1} S_{1}\left[\frac{\left(1-\vartheta_{1}\right) \Psi_{1} K_{L}}{\left(K_{11}+S_{1}\right)\left(K_{L}+S_{2}\right)}-\frac{\vartheta_{2} \Psi_{2} S_{2}}{\left(K_{21}+S_{1}\right)\left(K_{22}+S_{2}\right)}\right], \quad \mathbf{X} \in \Omega^{*}
\end{aligned}
$$

\section{Mass balance for Veillonella}

$$
\begin{aligned}
& \frac{\partial \vartheta_{2}}{\partial T}+\nabla \chi \cdot \nabla \vartheta_{2} \\
& \quad=\vartheta_{2} S_{1}\left[\frac{\left(1-\vartheta_{2}\right) \Psi_{2} S_{2}}{\left(K_{21}+S_{1}\right)\left(K_{22}+S_{2}\right)}-\frac{\vartheta_{1} \Psi_{1} K_{L}}{\left(K_{11}+S_{1}\right)\left(K_{L}+S_{2}\right)}\right], \quad \mathbf{X} \in \Omega^{*} .
\end{aligned}
$$

\section{Potential equation}

$$
\nabla^{2} \chi=S_{1}\left[\frac{\vartheta_{1} \Psi_{1} K_{L}}{\left(K_{11}+S_{1}\right)\left(K_{L}+S_{2}\right)}+\frac{\vartheta_{2} \Psi_{2} S_{2}}{\left(K_{21}+S_{1}\right)\left(K_{22}+S_{2}\right)}\right], \quad \mathbf{X} \in \mathrm{B}_{\mathrm{t}}^{*} .
$$

\section{B Numerical Methods}

Two types of PDEs, namely the elliptic Eqs. (25) and (29) and the time dependent advection-diffusion-reaction (ADR) Eqs. (27) and (28) (or advection-reaction equations) are involved in the current biofilm model. The mass balance equations of the lactic acid and the biomass (S. gordonii and Veillonella) are solved simultaneously in this study. The Time-discontinuous Galerkin (TDG) method (Hughes and Hulbert 1988; Hulbert 1992) is applied for solving the time dependent PDEs. The finite increment calculus (FIC) method (Oñate 1998; Oñate et al. 2007) is used to stabilize the numerical solutions. We would like to refer to (Sapotnick and Nackenhorst 2012; Feng et al. 2017) for more detailed interpretation of the TDG-FIC method on solving the time dependent ADR equations. In this study, 4-node bi-linear iso-parametric elements are applied for spatial discretization of the mass balance equations of the saliva, lactic acid and biomass. 8-node second order iso-parametric elements are used for solving the potential equation. The free boundary (moving biofilm-fluid interface) at each time step is captured by using an iso-line of $80 \%$ of total biomass volume fraction. Detailed information on the numerical schemes involved in the numerical strategy will be presented in the following part. As a remark, only one and two- dimensional problems are studied in this paper even through the mathematical model also can be applied for three-dimensional problems.

\section{B.1 Finite Element Approximation of Nonlinear Poisson Equation with $C^{0}$ Elements}

Equations (25) and (29) are Poisson equations. In this study, they are solved by using conventional Galerkin finite element method with $C^{0}$ type of elements. Both of these 
two equations can be written generally as

$$
\begin{aligned}
-\nabla^{2} q & =f, \quad q \in \mathcal{B}, \\
q & =c, \quad q \in \Gamma_{D}, \\
\mathbf{n} \cdot \nabla q & =0, \quad q \in \Gamma_{N},
\end{aligned}
$$

where $q$ represents the primary variables as $S_{1}$ or $\chi$ in the governing equations. $f$ denotes a function as $f=-\Theta_{11}^{2} \vartheta_{1} \frac{S_{1} K_{L}}{\left(K_{11}+S_{1}\right)\left(K_{L}+S_{2}\right)}-\Theta_{12}^{2} \vartheta_{2} \frac{S_{1} S_{2}}{\left(K_{21}+S_{1}\right)\left(K_{22}+S_{2}\right)}$ for Eq. (25) and $f=-S_{1}\left[\frac{\vartheta_{1} \Psi_{1} K_{L}}{\left(K_{11}+S_{1}\right)\left(K_{L}+S_{2}\right)}+\frac{\vartheta_{2} \Psi_{2} S_{2}}{\left(K_{21}+S_{1}\right)\left(K_{22}+S_{2}\right)}\right]$ for Eq. (29). $\mathcal{B}$ refers to the computational domain with the corresponding Dirichlet and Neuman boundaries $\Gamma_{D}$ and $\Gamma_{N} . \mathbf{n}$ is the norm vector on the Neuman boundary and $c$ is a constant value corresponding to the Dirichlet boundary. The weak form of Eq. (30) can be written as

$$
a(q, w)=F(w), \quad \forall w \in \mathcal{V},
$$

where,

$$
\begin{aligned}
a(q, w) & =\int_{\mathcal{B}} \nabla q \nabla w \mathrm{~d} V, \\
F(w) & =\int_{\mathcal{B}} f w \mathrm{~d} V .
\end{aligned}
$$

$w$ is an arbitrary test function in space $\mathcal{V}$ which is a subspace of the Hilbert space $\mathcal{H}^{1}$. As a remark, the Newton-Raphson method (Wriggers 2008) is used when the equation is nonlinear.

\section{B.2 TDG-FIC Approximation of Time Dependent Advection-Diffusion-reaction Equation}

Equations (26)-(28) are time-dependent PDEs. Therefore, they are solved simultaneously by using the TDG-FIC method. Those three equations can be written as a set of time dependent advection-diffusion-reaction partial differential equations as

$$
\frac{\partial \boldsymbol{q}}{\partial t}+\tilde{\boldsymbol{u}}^{T} \tilde{\nabla} \boldsymbol{q}-\tilde{\nabla}^{T} \mathbf{B} \tilde{\nabla} \boldsymbol{q}-\boldsymbol{f}(\boldsymbol{q})=\mathbf{0}
$$

where $\boldsymbol{q}=\left(S_{2}, \vartheta_{1}, \vartheta_{2}\right)^{T}$ denotes the unknown vector. Detailed information on the matrix form of each term in Eq. (33) can be found in C.

Applying discontinuous Galerkin method for time discretization and standard Galerkin discretization in space yields a time-space weak form of Eq. (33) over a 
time-space domain $\Omega \times \mathcal{T}_{n}$ (see Figure 2 in Feng et al. (2019))

$$
\begin{aligned}
& \int_{\Omega} w \int_{\mathcal{T}_{n}} v\left(\frac{\partial \boldsymbol{q}^{h}}{\partial t}+\tilde{\boldsymbol{u}}^{T} \tilde{\nabla} \boldsymbol{q}^{h}-\tilde{\nabla}^{T} \tilde{\boldsymbol{D}} \tilde{\nabla} \boldsymbol{q}^{h}-\boldsymbol{f}\left(\boldsymbol{q}^{h}\right)\right) \mathrm{d} t \mathrm{~d} \Omega \\
& \quad+\int_{\Omega} w v_{n-1}^{+} \llbracket \boldsymbol{q}_{n-1}^{h} \rrbracket \mathrm{d} \Omega=0,
\end{aligned}
$$

where $\Omega$ and $\mathcal{T}_{n}$ denote the spatial and temporal computational domains, respectively. $\boldsymbol{q}^{h}$ denotes the time-space approximation of the variable vector. Since the nodal value at a time point is discontinuous, a jump value of $\boldsymbol{q}_{n-1}^{h}$ at $t_{n-1}$, which is notated as $\llbracket \boldsymbol{q}_{n-1}^{h} \rrbracket$, is introduced as

$$
\llbracket \boldsymbol{q}_{n-1}^{h} \rrbracket=\boldsymbol{q}_{n-1}^{h+}-\boldsymbol{q}_{n-1}^{h-} .
$$

Due to the non-linearity of the reaction terms $\boldsymbol{f}\left(\boldsymbol{q}^{h}\right)$, the Newton-Raphson method (Wriggers 2008) is applied and Eq. (34) is linearized by introducing an incremental process with $\boldsymbol{q}^{h, 0}=\mathbf{0}$ as

$$
\begin{aligned}
& \boldsymbol{q}^{h, \beta+1}=\boldsymbol{q}^{h, \beta}+\Delta \boldsymbol{q}^{h}, \\
& \boldsymbol{f}\left(\boldsymbol{q}^{h, \beta+1}\right) \approx \boldsymbol{f}\left(\boldsymbol{q}^{h, \beta}\right)+\left.\frac{\partial \boldsymbol{f}}{\partial \boldsymbol{q}}\right|_{\boldsymbol{q}^{h, \beta}} \Delta \boldsymbol{q}^{h} .
\end{aligned}
$$

Substituting (36) into (34) yields the linearized time-space weak form

$$
\begin{gathered}
\int_{\Omega} w \int_{\mathcal{T}_{n}} v\left(\frac{\partial \Delta \boldsymbol{q}^{h}}{\partial t}+\tilde{\boldsymbol{u}}^{T} \tilde{\boldsymbol{\nabla}}\left(\Delta \boldsymbol{q}^{h}\right)-\tilde{\nabla}^{T} \tilde{\boldsymbol{D}} \tilde{\nabla}\left(\Delta \boldsymbol{q}^{h}\right)-\left.\frac{\partial \boldsymbol{f}}{\partial \boldsymbol{q}}\right|_{\boldsymbol{q}^{h, \beta}} \Delta \boldsymbol{q}^{h}\right) \mathrm{d} t \mathrm{~d} \Omega \\
+\int_{\Omega} w v_{n-1}^{+} \Delta \boldsymbol{q}_{n-1}^{h+} \mathrm{d} \Omega=\int_{\Omega} w v_{n+1}^{+} \boldsymbol{q}_{n-1}^{h-, \beta} \mathrm{d} \Omega-\int_{\Omega} w v_{n-1}^{+} \boldsymbol{q}_{n-1}^{h+, \beta} \mathrm{d} \Omega \\
-\int_{\Omega} w \int_{\mathcal{T}_{n}} v\left(\frac{\partial \boldsymbol{q}^{h, \beta}}{\partial t}+\tilde{\boldsymbol{u}}^{T} \tilde{\nabla} \boldsymbol{q}^{h, \beta}-\tilde{\nabla}^{T} \tilde{\boldsymbol{D}} \tilde{\nabla} \boldsymbol{q}^{h, \beta}-\boldsymbol{f}\left(\boldsymbol{q}^{h, \beta}\right)\right) \mathrm{d} t \mathrm{~d} \Omega .
\end{gathered}
$$

The unknown field of $\boldsymbol{q}^{h}$ is interpolated over the time-space space by

$$
\boldsymbol{q}^{h}=\sum_{k=1}^{n_{t}} \hat{N}_{k} \sum_{j=1}^{n_{n}} \tilde{N}_{j} \hat{\tilde{\boldsymbol{q}}}_{j k}
$$

where $n_{t}$ and $n_{n}$ refer to temporal and spatial total degrees of freedoms respectively. $\hat{N}$ and $\tilde{N}$ are the temporal and spatial shape functions and $\hat{\tilde{\boldsymbol{q}}}$ denotes the time-space nodal variable vector. 
Discretization of Eq. (37) in space and time by adopting Eq. (38) within single time-space element $\Omega^{e} \times \mathcal{T}_{n}$ yields

$$
\begin{aligned}
& {\left[\left(\tilde{\boldsymbol{N}}^{T}, \tilde{\boldsymbol{N}}\right)^{e} \otimes \mathbf{T}_{a}^{n}\right.} \\
& \left.\quad+\left(\left(-\tilde{\boldsymbol{B}}^{T} \mathbf{Q}, \tilde{\boldsymbol{N}}\right)^{e}+\left(\tilde{\boldsymbol{B}}^{T} \tilde{\boldsymbol{D}}, \tilde{\boldsymbol{B}}\right)^{e}-\left(\left.\tilde{\boldsymbol{N}}^{T} \frac{\partial \boldsymbol{f}}{\partial \boldsymbol{q}}\right|_{\boldsymbol{q}^{h, \beta}}, \tilde{\boldsymbol{N}}\right)^{e}\right) \otimes \mathbf{T}_{b}^{n}\right] \Delta \hat{\tilde{\boldsymbol{q}}} \\
& =\left(\hat{\tilde{\boldsymbol{q}}}_{n-1}^{-}, \tilde{\boldsymbol{N}}^{T}\right)^{e} \otimes \mathbf{T}_{c}^{n}-\left(\tilde{\boldsymbol{N}}^{T}, \tilde{\boldsymbol{N}}\right)^{e} \otimes \mathbf{T}_{a}^{n} \hat{\tilde{\boldsymbol{q}}}^{\beta} \\
& \quad-\left[\left(-\tilde{\boldsymbol{B}}^{T} \mathbf{Q}, \tilde{\boldsymbol{N}}\right)^{e} \hat{\tilde{\boldsymbol{q}}}^{\beta}+\left(\tilde{\boldsymbol{B}}^{T} \tilde{\boldsymbol{D}}, \tilde{\boldsymbol{B}}\right)^{e} \hat{\tilde{\boldsymbol{q}}}^{\beta}-\left(\boldsymbol{f}\left(\hat{\tilde{\boldsymbol{q}}}^{\beta}\right), \tilde{\boldsymbol{N}}^{T}\right)^{e}\right] \otimes \mathbf{T}_{b}^{n},
\end{aligned}
$$

where

$$
(m, n)^{e}=\int_{\Omega^{e}} m m \mathrm{~d} \Omega,
$$

$\hat{\tilde{\boldsymbol{q}}}$ refers to the time-space approximation of the nodal variables. $\mathbf{T}_{a}, \mathbf{T}_{b}, \mathbf{T}_{c}$ are time matrixes with only temporal shape function $\hat{N}$ involved

$$
\begin{aligned}
\mathbf{T}_{a} & =\int_{\mathcal{T}_{n}} \hat{\boldsymbol{N}}^{T} \frac{\mathrm{d} \hat{\boldsymbol{N}}}{\mathrm{d} t} \mathrm{~d} t+\left(\hat{\boldsymbol{N}}_{n-1}^{+}\right)^{T} \hat{\boldsymbol{N}}_{n-1}^{+}, \\
\mathbf{T}_{b} & =\int_{\mathcal{T}_{n}} \hat{\boldsymbol{N}}^{T} \hat{\boldsymbol{N}} \mathrm{d} t \\
\mathbf{T}_{c} & =\left(\hat{\boldsymbol{N}}_{n-1}^{+}\right)^{T} \hat{\boldsymbol{N}}_{n-1}^{-} .
\end{aligned}
$$

$\tilde{N}$ is extended spatial shape function depending on the spatial element type (see C for detailed matrix form of $\tilde{N}$ ).

The diffusion term in (33) only affects the mass balance equation of the lactic acid and does not appear in the mass balance equations of biomass. Therefore, the system of the mass balance equations in this model is still hyperbolic dominated. It is well known that solving hyperbolic PDEs with the conventional Galerkin method suffers from convective instability (Donea and Huerta 2003). One way to relieve such a problem is by adding an additional artificial diffusion (balancing diffusion) term into the system. This yields the so-called "stabilization methods" (Codina 1998; Franca et al. 1992; Lian et al. 2016) which have been developed for Galerkin methods over the past decades. In this paper, one of the widely used stabilization methods, namely the finite increment calculus (FIC) method, which introduces a nonlinear balancing artificial diffusion term in (39) is adopted. Thus, the diffusion coefficient matrix $\tilde{\boldsymbol{D}}$ is replaced by

$$
\tilde{\boldsymbol{D}}^{S T B}=\tilde{\boldsymbol{D}}+\tilde{\boldsymbol{D}}_{F I C}
$$

where

$$
\tilde{\boldsymbol{D}}_{F I C}=\left(\begin{array}{ccc}
\boldsymbol{D}_{I}^{*}\left(S_{2}\right) & \mathbf{0} & \mathbf{0} \\
\mathbf{0} & \boldsymbol{D}_{\boldsymbol{I} I}^{*}\left(\vartheta_{1}\right) & \mathbf{0} \\
\mathbf{0} & \mathbf{0} & \boldsymbol{D}_{\boldsymbol{I I I}}^{*}\left(\vartheta_{2}\right)
\end{array}\right)
$$


$\boldsymbol{D}_{\boldsymbol{I}}^{*}, \boldsymbol{D}_{\boldsymbol{I} \boldsymbol{I}}^{*}$ and $\boldsymbol{D}_{\boldsymbol{I I I} \boldsymbol{I}}^{*}$ are $2 \times 2$ diagonal matrices depending on the variables $S_{2}, \vartheta_{1}$ and $\vartheta_{2}$ respectively as in (43). Detailed calculation procedures of these $\boldsymbol{D}^{*}$ matrices for multi-dimensional problems can be found in (Feng et al. 2017). As a remark, $\tilde{\boldsymbol{D}}_{\text {FIC }}$ depends on the solution of each spatial element at the current time step. Moreover, the artificial balancing diffusion is introduced along the directions of the principle curvatures of the solution and thus it must be calculated in a local coordinate system and then transferred to the global Cartesian coordinate system afterwards. Therefore, the FIC stabilized algorithm becomes nonlinear and iterations at each time step are required even if a linear problem is considered.

\section{Matrices in the Advection-Reaction Equation}

For two-dimensional problems, the advection and diffusion coefficients matrices read

$$
\begin{aligned}
& \tilde{\boldsymbol{u}}=\mathbf{I}_{3 \times 3} \otimes \mathbf{u}=\left(\begin{array}{lll}
1 & 0 & 0 \\
0 & 1 & 0 \\
0 & 0 & 1
\end{array}\right) \otimes\left(\begin{array}{l}
u_{x} \\
u_{y}
\end{array}\right)=\left(\begin{array}{ccc}
u_{x} & 0 & 0 \\
u_{y} & 0 & 0 \\
0 & u_{x} & 0 \\
0 & u_{y} & 0 \\
0 & 0 & u_{x} \\
0 & 0 & u_{y}
\end{array}\right), \\
& \tilde{\nabla}=\mathbf{I}_{3 \times 3} \otimes \nabla \\
& \otimes
\end{aligned}
$$

where $u_{x}$ and $u_{y}$ are the advection velocity components along $x$ and $y$ coordinates respectively. I denotes an identity matrix and $\otimes$ is the Kronecker product. I denotes an identical matrix and $\otimes$ is the Kronecker product. $\boldsymbol{D}_{2}^{*}$ denotes a $2 \times 2$ diagonal matrix with $D_{2}^{*}$ placed at the main diagonal and $\mathbf{0}$ denotes a $2 \times 2$ null matrix. Furthermore, $f(q)$ reads

$$
\boldsymbol{f}(\boldsymbol{q})=\left(f_{1}\left(S_{2}, \vartheta_{1}, \vartheta_{2}\right), f_{2}\left(S_{2}, \vartheta_{1}, \vartheta_{2}\right), f_{3}\left(S_{2}, \vartheta_{1}, \vartheta_{2}\right)\right)^{T}
$$

where $f_{1}, f_{2}$ and $f_{3}$ denote the reaction terms of Eqs. (26)-(28) respectively.

For four-node $2 \mathrm{D}$ bi-linear element, the shape function is extended to

$$
\tilde{N}=N \otimes \mathbf{I}_{3 \times 3},
$$


where $\boldsymbol{N}=\left[N_{1}, N_{2}, N_{3}, N_{4}\right]$ is the shape function of the four-node $2 \mathrm{D}$ bi-linear element. Similarly,

$$
\tilde{\boldsymbol{B}}=\boldsymbol{B} \otimes \mathbf{I}_{3 \times 3}
$$

\section{References}

Alpkvist E, Klapper I (2007) A multidimensional multispecies continuum model for heterogeneous biofilm development. Bull Math Biol 69(2):765-789

Bradshaw DJ, Marsh PD (1998) Analysis of pH-driven disruption of oral microbial communities in vitro. Caries Res 32(6):456-462

Chalmers NI (2008) Multispecies oral biofilms studied at the single community level as a model system for spatiotemporal development of biofilms and interspecies interactions. Ph.d. thesis, University of Maryland, Baltimore, Maryland, United States

Chalmers NI, Palmer RJ, Cisar JO, Kolenbrander PE (2008) Characterization of a Streptococcus sp.-Veillonella $s p$. community micromanipulated from dental plaque. Journal of Bacteriology 190(24):8145-8154

Chihara K, Matsumoto S, Kagawa Y, Tsuneda S (2015) Mathematical modeling of dormant cell formation in growing biofilm. Front Microbiol 6:534

Codina R (1998) Comparison of some finite element methods for solving the diffusion-convection-reaction equation. Comput Methods Appl Mech Eng 156(1):185-210

Cogan NG (2004) The role of the biofilm matrix in structural development. Math Med Biol 21(2):147-166

Corbin A, Pitts B, Parker A, Stewart PS (2011) Antimicrobial penetration and efficacy in an in vitro oral biofilm model. Antimicrob Agents Chemoth 55(7):3338-3344

Donea J, Huerta A (2003) Finite element methods for flow problems. Wiley, New York

Duddu R, Chopp DL, Moran B (2009) A two-dimensional continuum model of biofilm growth incorporating fluid flow and shear stress based detachment. Biotechnol Bioeng 103(1):92-104

Eberl HJ, Parker DF, van Loosdrecht Mark C M (2001) A new deterministic spatio-temporal continuum model for biofilm development. J Theor Med 3(3):161-175

Egland PG, Palmer RJ, Kolenbrander PE (2004) Interspecies communication in Streptococcus gordoniiVeillonella atypica biofilms: signaling in flow conditions requires juxtaposition. Proc Natl Acad Sci 101(48):16917-16922

Emerenini BO, Hense BA, Kuttler C, Eberl HJ (2015) A mathematical model of quorum sensing induced biofilm detachment. PLOS One 10(7):1-25

Feng D, Neuweiler I, Nackenhorst U (2017) A spatially stabilized TDG based finite element framework for modeling biofilm growth with a multi-dimensional multi-species continuum biofilm model. Comput Mech 59(6):1049-1070

Feng D, Rath H, Neuweiler I, Stumpp N, Nackenhorst U, Stiesch M (2018) A deeper insight of a multi-dimensional continuum biofilm growth model: experimental observation and parameter studies, experiments and simulation. In: Wriggers P, Lenarz T (eds) Biomedical technology: modeling. Springer International Publishing, Cambridge, pp 257-272

Feng D, Neuweiler I, Nackenhorst U, Wick T (2019) A time-space flux-corrected transport finite element formulation for solving multi-dimensional advection-diffusion-reaction equations. J Comput Phys 396:31-53

Franca LP, Frey SL, Hughes TJ (1992) Stabilized finite element methods: I. Application to the advectivediffusive model. Comput Methods Appl Mech Eng 95(2):253-276

Fujikawa H (1994) Diversity of the growth patterns of Bacillus subtilis colonies on agar plates. FEMS Microbiol Ecol 13(3):159-168

Ghasemi M, Hense BA, Eberl HJ, Kuttler C (2018) Simulation-based exploration of quorum sensing triggered resistance of biofilms to antibiotics. Bull Math Biol 80(7):1736-1775

Gordeeva YL, Rudakovskaya E, Gordeeva E, Borodkin A (2017) Mathematical modeling of biotechnological process of lactic acid production by batch fermentation: a review. Theor Found Chem Eng 51(3):282-298

Hughes TJ, Hulbert GM (1988) Space-time finite element methods for elastodynamics: formulations and error estimates. Comput Methods Appl Mech Eng 66(3):339-363 
Hulbert GM (1992) Time finite element methods for structural dynamics. Int J Numer Methods Eng 33(2):307-331

Kara D, Luppens SBI, van Marle J, Ozok R, ten Cate JM (2007) Microstructural differences between singlespecies and dual-species biofilms of Streptococcus mutans and Veillonella parvula, before and after exposure to chlorhexidine. FEMS Microbiol Lett 271(1):90-97

Khassehkhan H, Eberl HJ (2008) Modeling and simulation of a bacterial biofilm that is controlled by ph and protonated lactic acids. Comput Math Methods Med 9(1):47-67

Klapper I, Dockery J (2002) Finger formation in biofilm layers. SIAM J Appl Math 62(3):853-869

Kolenbrander PE, Palmer RJJR, Periasamy S, Jakubovics NS (2010) Oral multispecies biofilm development and the key role of cell-cell distance. Nat Rev Microbiol 8(7):471-480

Kommerein N, Stumpp SN, Müsken M, Ehlert N, Winkel A, Häussler S, Behrens P, Buettner FFR, Stiesch M (2017) An oral multispecies biofilm model for high content screening applications. PloS One 12(3):e0173973

Kreft JU, Picioreanu C, Wimpenny JW, van Loosdrecht MC (2001) Individual-based modelling of biofilms. Microbiology (Reading, England) 147(Pt 11):2897-2912

Lian Y, Ying Y, Tang S, Lin S, Wagner GJ, Liu WK (2016) A Petrov-Galerkin finite element method for the fractional advection-diffusion equation. Comput Methods Appl Mech Eng 309:388-410

Lindley B, Wang Q, Zhang T (2012) Multicomponent hydrodynamic model for heterogeneous biofilms: two-dimensional numerical simulations of growth and interaction with flows. Physical Review E, Statistical, Nonlinear, and Soft Matter Physics 85(3 Pt 1):031908

Madigan MT (2012) Brock biology of microorganisms, 13th edn. Benjamin Cummings, San Francisco

Martin B, Tamanai-Shacoori Z, Bronsard J, Ginguené F, Meuric V, Mahé F, Bonnaure-Mallet M (2017) A new mathematical model of bacterial interactions in two-species oral biofilms. PloS One 12(3):e0173153

Martin KJ, Picioreanu C, Nerenberg R (2015) Assessing microbial competition in a hydrogen-based membrane biofilm reactor (MBfR) using multidimensional modeling. Biotechnol Bioeng 112(9):18431853

Mashima I, Nakazawa F (2015) The interaction between Streptococcus spp. and Veillonella tobetsuensis in the early stages of oral biofilm formation. J Bacteriol 197:2104

Mattei MR, Frunzo L, DAcunto B, Pechaud Y, Pirozzi F, Esposito G (2017) Continuum and discrete approach in modeling biofilm development and structure: a review. J Math Biol 76:945

Nascimento MM, Gordan VV, Garvan CW, Browngardt CM, Burne RA (2009) Correlations of oral bacterial arginine and urea catabolism with caries experience. Oral Microbiol Immunol 24(2):89-95

Noguera D, Pizarro G, Stahl D, Rittmann B (1999) Simulation of multispecies biofilm development in three dimensions. Water Sci Technol 39(7):123-130

Oñate E (1998) Derivation of stabilized equations for numerical solution of advective-diffusive transport and fluid flow problems. Comput Methods Appl Mech Eng 151(1-2):233-265

Oñate E, Miquel J, Zárate F (2007) Stabilized solution of the multidimensional advection-diffusionabsorption equation using linear finite elements. Comput Fluids 36(1):92-112

Paquette DW, Brodala N, Williams RC (2006) Risk factors for endosseous dental implant failure. Dent Clin N Am 50(3):361-74

Periasamy S, Kolenbrander PE (2010) Central role of the early colonizer Veillonella sp in establishing multispecies biofilm communities with initial, middle, and late colonizers of enamel. J Bacteriol 192(12):2965-2972

Picioreanu C, van Loosdrecht MC, Heijnen JJ (1998) A new combined differential-discrete cellular automaton approach for biofilm modeling: application for growth in gel beads. Biotechnol Bioeng 57(6):718-731

Picioreanu C, van Loosdrecht MC, Heijnen JJ (2000) Effect of diffusive and convective substrate transport on biofilm structure formation: a two-dimensional modeling study. Biotechnol Bioeng 69(5):504-515

Picioreanu C, Kreft JU, van Loosdrecht MCM (2004) Particle-based multidimensional multispecies biofilm model. Appl Environ Microbiol 70(5):3024-3040

Picioreanu C, Rittmann B, Van Loosdrecht M (2006) Mathematical modelling of biofilms. IWA Publishing, London, UK

Rahman KA, Sudarsan R, Eberl HJ (2015) A mixed-culture biofilm model with cross-diffusion. Bull Math Biol 77(11):2086-2124 
Rath H, Feng D, Neuweiler I, Stumpp NS, Nackenhorst U, Stiesch M (2017) Biofilm formation by the oral pioneer colonizer Streptococcus gordonii: an experimental and numerical study. FEMS Microbiol Ecol 93:3

Ribeiro ACF, Lobo VMM, Leaist DG, Natividade JJS, Veríssimo LP, Barros MCF, Cabral AMTDPV (2005) Binary diffusion coefficients for aqueous solutions of lactic acid. J Solut Chem 34(9):1009-1016

Rittmann BE, McCarty PL (1980) Model of steady-state-biofilm kinetics. Biotechnol Bioeng 22(11):23432357

Sapotnick A, Nackenhorst U (2012) A combined FIC-TDG finite element approach for the numerical solution of coupled advection-diffusion-reaction equations with application to a bioregulatory model for bone fracture healing. Int J Numer Methods Eng 92(3):301-317

Tang Y, Liu H (2017) Modeling multidimensional and multispecies biofilms in porous media. Biotechnol Bioeng 114(8):1679-1687

Tang Y, Valocchi AJ (2013) An improved cellular automaton method to model multispecies biofilms. Water Res 47(15):5729-5742

Wanner O, Gujer W (1986) A multispecies biofilm model. Biotechnol Bioeng 28(3):314-328

Wanner O, Reichert P (1996) Mathematical modeling of mixed-culture biofilms. Biotechnol Bioeng 49(2):172-184

Wellmann C, Wriggers P (2012) A two-scale model of granular materials. Comput Methods Appl Mech Eng 205-208:46-58

Wimpenny JW, Colasanti R (1997) A unifying hypothesis for the structure of microbial biofilms based on cellular automaton models. FEMS Microbiol Ecol 22(1):1-16

Wriggers P (2008) Nonlinear finite element methods. Springer, Berlin

Xavier JB, Picioreanu C, Van Loosdrecht MC (2005) A framework for multidimensional modelling of activity and structure of multispecies biofilms. Environ Microbiol 7(8):1085-1103

Yang L, Liu Y, Wu H, Hóiby N, Molin S, Song Z (2011) Current understanding of multi-species biofilms. Int J Oral Sci 3(2):74-81

Zhang T, Cogan N, Wang Q (2008a) Phase field models for biofilms. II. 2-D numerical simulations of biofilm-flow interaction. Commun Comput Phys 4(1):72-101

Zhang T, Cogan NG, Wang Q (2008b) Phase field models for biofilms. I. Theory and one-dimensional simulations. SIAM J Appl Math 69(3):641-669

Zhao J, Wang Q (2017) Three-dimensional numerical simulations of biofilm dynamics with Quorum Sensing in a flow cell. Bull Math Biol 79(4):884-919

Publisher's Note Springer Nature remains neutral with regard to jurisdictional claims in published maps and institutional affiliations. 